\title{
The Modulatory Effect of Motor Cortex Astrocytes on Diabetic Neuropathic Pain
}

\author{
Jingshan Lu, ${ }^{1 *}$ Lan Yang, ${ }^{1 *}$ Ying Xu, ${ }^{1 *}$ Lijing Ai, ${ }^{1 *}$ Jian Chen, ${ }^{1}$ Fangfang Xiong, ${ }^{1}$ Lihuan Hu, ${ }^{1}$ Huoshu Chen, ${ }^{1}$ \\ Jiyuan Liu, ${ }^{2}$ Xiongbin Yan, ${ }^{2}$ Huihui Huang, ${ }^{1}$ Li Chen, ${ }^{1,3}$ and Changxi $\mathbf{Y u}^{1,3}$ \\ ${ }^{1}$ Department of Pharmacology, School of Pharmacy, Fujian Medical University, Fuzhou, Fujian 350122, China, ${ }^{2}$ School of Clinical Medicine, Fujian \\ Medical University, Fuzhou, Fujian 350122, China, and ${ }^{3}$ Fujian Key Laboratory of Drug Target Discovery and Structural and Functional Research, \\ Fujian Medical University, Fuzhou, Fujian 350122, China
}

Diabetic neuropathic pain (DNP) is a common complication of diabetes characterized by persistent pain. Emerging evidence links astrocytes to mechanical nociceptive processing, and the motor cortex (MCx) is a cerebral cortex region that is known to play a key role in pain regulation. However, the association between $\mathrm{MCx}$ astrocytes and DNP pathogenesis remains largely unexplored. Here, we studied this association using designer receptors exclusively activated by designer drugs to specifically manipulate MCx astrocytes. We proved that the selective inhibition of MCx astrocytes reduced DNP in streptozocin (STZ)induced DNP models and discovered a potential mechanism by which astrocytes release cytokines, including TNF- $\alpha$ and IL$1 \beta$, to increase neuronal activation in the $\mathrm{MCx}$, thereby regulating pain. Together, these results demonstrate a pivotal role for $\mathrm{MCx}$ astrocytes in DNP pathogenesis and provide new insight into DNP treatment strategies.

Key words: astrocytes; diabetic neuropathic pain; DREADDs; motor cortex

Significance Statement

Astrocytes are critical for maintaining CNS homeostasis. In recent years, astrocytes have been demonstrated to play roles in pain signaling modulation and neuropathic pain maintenance, with studies showing that they inhibit pain transmission at the spinal level. This work suggests that astrocytes also modulate pain at the supraspinal level. Indeed, we show that chemogenetically manipulated MCx astrocytes affect the mechanical withdrawal thresholds of rats and elucidate a potential mechanism by which astrocytes release inflammatory mediators to increase neuronal activation in the $\mathrm{MCx}$, thereby regulating pain. Together, our data support that inhibition of astrocytes in the $\mathrm{MCx}$ region might have broad prospects for diabetic neuropathic pain treatment.

\section{Introduction}

Diabetic neuropathic pain (DNP) is a common chronic complication of diabetes mellitus characterized by disordered pain sensation, displayed as spontaneous pain, allodynia, and hyperalgesia (Peltier et al., 2014). Approximately $30 \%$ of patients with diabetes reportedly

Received Oct. 3, 2020; revised Feb. 11, 2021; accepted Mar. 11, 2021.

Author contributions: J. Lu, L.Y., L.A., J.C., F.X., L.H., H.C., J. Liu, X.Y., and H.H. performed research; J. Lu, L.Y., and Y.X. analyzed data; J. Lu, L.Y., and L.A. wrote the first draft of the paper; J. Lu, L.A., Y.X., L.C., and C.Y. edited the paper; J. Lu wrote the paper; Y.X., L.C., and C.Y. designed research.

This work was supported by National Natural Science Foundation of China Grants 81773716 and 81973309 to C.Y. and Grant 81701307 to L.C., Joint Funds for the Innovation of Science and Technology, Fujian Province Grant 2016 Y9058 to C.Y., Special Support Funds for the Science and Technology Innovation Leader, Fujian Province Grant 2016B017 to C.Y., Drug Innovation Major Project of China Grant 2018ZX09711001-003-024 to C.Y., Industry-University-Research Cooperation Project, Fujian Province Grant 2017Y4007 to C.Y., and Fujian Medical University Startup Fund for Scientific Research Grant 2018QH2017 to J.Lu.

*J.Lu, L.Y., Y.X., and L.A. Y.X. contributed equally to this work.

The authors declare no competing financial interests.

Correspondence should be addressed to Changxi Yu at changxiyu@mail.jmu.edu.cn or Li Chen at lichen@fjmu.edu.cn.

https://doi.org/10.1523/JNEUROSCI.2566-20.2021

Copyright $\odot 2021$ the authors suffer from DNP, but there is a paucity of treatments (Feldman et al., 2019). Current therapeutic strategies for DNP are mostly symptomatic and thus primarily concentrate on pain management (Bönhof et al., 2019; Alam et al., 2020). However, most analgesics have been demonstrated to provide only partial pain relief or have multiple side effects (Rosenberger et al., 2020). For example, as a potent opioid analgesic, morphine significantly prolongs the duration of hyperalgesia in diabetic rodents (Grace et al., 2016). This problematic feature of the current DNP therapeutic strategies highlights the urgent need to advance our understanding of the DNP mechanism and to identify novel therapeutic targets.

As previous clinical and preclinical studies centering merely on neurons failed to account for the complexity of DNP pathogenesis, studies on astrogliopathy in the CNS may uncover novel targets for neuropathic therapies (Ishikawa et al., 2018; Ji et al., 2019; Rajchgot et al., 2019). Astrocytes, accounting for $20 \%-40 \%$ of all glial cells in the CNS, are the largest nonneuronal cells in the CNS, and a series of studies has consistently verified that astrocytes play a pivotal role in the manipulation of DNP (Ji et al., 2016; Li et al., 2019). However, while the current focus 
Table 1. Statistical analyses in each figure

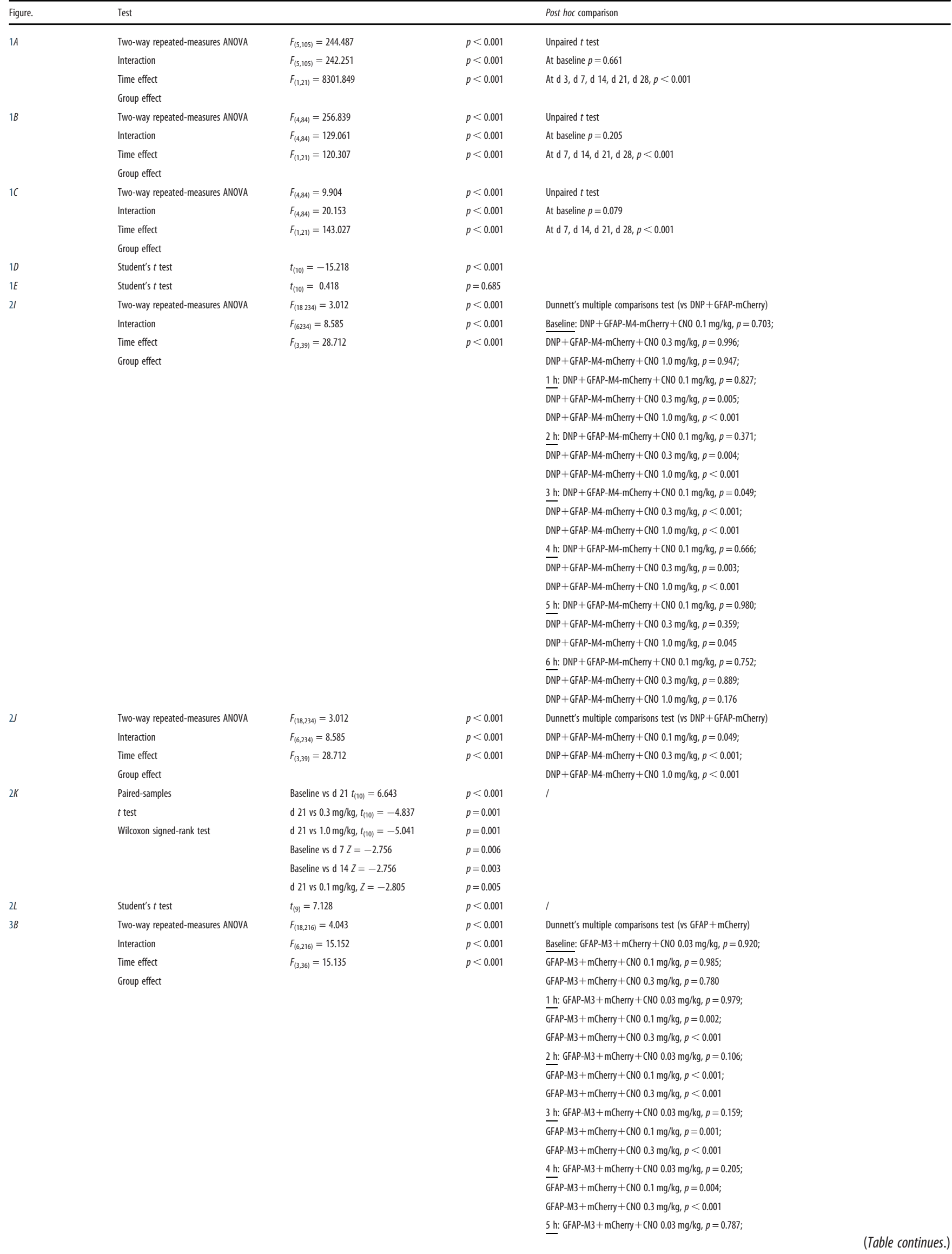


Table 1. Continued

\begin{tabular}{|c|c|c|c|c|}
\hline Figure. & Test & & & Post hoc comparison \\
\hline & & & & GFAP-M3+mCherry+CN0 $0.1 \mathrm{mg} / \mathrm{kg}, p=0.894$ \\
\hline & & & & GFAP-M3 + mCherry + CN0 $0.3 \mathrm{mg} / \mathrm{kg}, p=0.068$ \\
\hline & & & & 6 h: GFAP-M3 + mCherry + CNO $0.03 \mathrm{mg} / \mathrm{kg}, p=0.856$; \\
\hline & & & & GFAP-M3 + mCherry+CN0 $0.1 \mathrm{mg} / \mathrm{kg}, p=0.872$ \\
\hline & & & & GFAP-M3 + mCherry + CNO $0.3 \mathrm{mg} / \mathrm{kg}, p=0.711$ \\
\hline \multirow[t]{4}{*}{$3 C$} & Two-way repeated-measures ANOVA & $F_{(18,216)}=4.043$ & $p<0.001$ & Dunnett's multiple comparisons test (vs GFAP+mCherry). \\
\hline & Interaction & $F_{(6,216)}=15.152$ & $p<0.001$ & GFAP-M3 + mCherry+CN0 0.03 mg $/ \mathrm{kg}, p=0.106$ \\
\hline & Time effect & $F_{(3,36)}=15.135$ & $p<0.001$ & GFAP-M3 + mCherry+CN0 $0.1 \mathrm{mg} / \mathrm{kg}, p<0.001$ \\
\hline & Group effect & & & GFAP-M3+mCherry+CN0 $0.3 \mathrm{mg} / \mathrm{kg}, p<0.001$ \\
\hline \multirow[t]{3}{*}{30} & Paired-samples & Baseline vs $d 22 t_{(11)}=4.5$ & $p=0.001$ & \\
\hline & $t$ test & Baseline vs $d 25 t_{(11)}=5.94$ & $p<0.001$ & \\
\hline & & Baseline vs d $28 t_{(11)}=9.594$ & $p<0.001$ & \\
\hline $3 E$ & Student's $t$ test & $t_{(8)}=-13.031$ & $p<0.001$ & \\
\hline \multirow[t]{6}{*}{$4 B$} & One-way ANOVA & $F_{(5,27)}=27.601$ & $p<0.001$ & Games-Howell multiple comparisons test \\
\hline & & & & Control vs DNP $p=0.005$ \\
\hline & & & & DNP + GFAP-M4-mCherry + CNO vs DNP $p=0.007$ \\
\hline & & & & DNP + GFAP-mCherry vs DNP+GFAP-M4-mCherry $p<0.001$ \\
\hline & & & & GFAP-M3 + mCherry vs DNP $p=1.000$ \\
\hline & & & & GFAP-mCherry vs GFAP-M3 + mCherry $p=0.017$ \\
\hline \multirow[t]{20}{*}{$5 E$} & Two-way repeated-measures ANOVA & $F_{(18,252)}=4.058$ & $p<0.001$ & Dunnett's multiple comparisons test (vs DNP + CaMKII-mCherry) \\
\hline & Interaction & $F_{(6,252)}=16.851$ & $p<0.001$ & Baseline: DNP+CaMKII-M4-mCherry+CNO 0.03 mg/kg, p=0.584; \\
\hline & Time effect & $F_{(3,42)}=80.494$ & $p<0.001$ & DNP + CaMKIII-M4-mCherry+CN0 $0.1 \mathrm{mg} / \mathrm{kg}, p=0.554$ \\
\hline & Group effect & & & DNP + CaMKII-M4-mCherry + CN0 $0.3 \mathrm{mg} / \mathrm{kg}, p=0.373$ \\
\hline & & & & 1 h: DNP+CaMKII-M4-mCherry+CN0 $0.03 \mathrm{mg} / \mathrm{kg}, p=0.152 ;$ \\
\hline & & & & $\overline{\mathrm{DNP}}+$ CaMKII-M4-mCherry + CN0 $0.1 \mathrm{mg} / \mathrm{kg}, p<0.001$ \\
\hline & & & & DNP+CaMKII-M4-mCherry + CN0 0.3 mg/kg, $p<0.001$ \\
\hline & & & & $2 \mathrm{~h}$ : DNP+CaMKII-M4-mCherry + CN0 $0.03 \mathrm{mg} / \mathrm{kg}, p=0.309 ;$ \\
\hline & & & & DNP+CaMKIII-M4-mCherry + CN0 $0.1 \mathrm{mg} / \mathrm{kg}, p<0.001$ \\
\hline & & & & DNP + CaMKII-M4-mCherry+ CN0 $0.3 \mathrm{mg} / \mathrm{kg}, p<0.001$ \\
\hline & & & & $3 \mathrm{~h}:$ DNP+CaMKII-M4-mCherry+CN0 0.03 mg/kg, $p=0.249 ;$ \\
\hline & & & & DNP+CaMKII-M4-mCherry + CNO $0.1 \mathrm{mg} / \mathrm{kg}, p<0.001 ;$ DNP + CaMKII-M4-mCherry+CNO $0.3 \mathrm{mg} / \mathrm{kg}, p<0.001$ \\
\hline & & & & 4 h: DNP+CaMKII-M4-mCherry + CN0 0.03 mg $/ \mathrm{kg}, p=0.228$ \\
\hline & & & & DNP+CaMKII-M4-mCherry+CN0 $0.1 \mathrm{mg} / \mathrm{kg}, p<0.001 ;$ DNP + CaMKII-M4-mCherry+CN0 $0.3 \mathrm{mg} / \mathrm{kg}, p<0.001$ \\
\hline & & & & $5 \mathrm{~h}:$ DNP + CaMKII-M4-mCherry + CNO $0.03 \mathrm{mg} / \mathrm{kg}, p=0.463$ \\
\hline & & & & $\overline{\mathrm{DNP}}+$ CaMKII-M4-mCherry + CN0 $0.1 \mathrm{mg} / \mathrm{kg}, p=0.011$ \\
\hline & & & & DNP + CaMKII-M4-mCherry + CN0 $0.3 \mathrm{mg} / \mathrm{kg}, p<0.001$ \\
\hline & & & & 6 h: DNP+CaMKII-M4-mCherry + CNO $0.03 \mathrm{mg} / \mathrm{kg}, p=0.792 ;$ \\
\hline & & & & $\overline{\mathrm{DNP}}+$ CaMKII-M4-mCherry + CNO $0.1 \mathrm{mg} / \mathrm{kg}, p=0.130$ \\
\hline & & & & DNP+CaMKII-M4-mCherry + CN0 $0.3 \mathrm{mg} / \mathrm{kg}, p=0.431$ \\
\hline \multirow[t]{4}{*}{$5 F$} & Two-way repeated-measures ANOVA & $F_{(18,252)}=4.058$ & $p<0.001$ & Dunnett's multiple comparisons test (vs DNP + CaMKII-mCherry) \\
\hline & Interaction & $F_{(6,252)}=16.851$ & $p<0.001$ & DNP+CaMKII-M4-mCherry+CNO $0.03 \mathrm{mg} / \mathrm{kg}, p=0.249$ \\
\hline & Time effect & $F_{(3,42)}=80.494$ & $p<0.001$ & DNP+CaMKII-M4-mCherry + CN0 0.1 mg/kg, $p<0.001 ;$ DNP + CaMKII-M4-mCherry+CNO $0.3 \mathrm{mg} / \mathrm{kg}, p<0.001$ \\
\hline & Group effect & & & \\
\hline \multirow[t]{6}{*}{$5 G$} & Paired-samples & Baseline vs $d 7 t_{(11)}=3.522$ & $p=0.005$ & l \\
\hline & $t$ test & Baseline vs $d 14 t_{(11)}=6.204$ & $p<0.001$ & \\
\hline & & Baseline vs $d 21 t_{(11)}=10.724$ & $p<0.001$ & \\
\hline & & $\mathrm{d} 21: 0.03 \mathrm{mg} / \mathrm{kg}, t_{(11)}=-1.07$ & $p=0.307$ & \\
\hline & & $\mathrm{d} 21: 0.1 \mathrm{mg} / \mathrm{kg}, t_{(11)}=-8.141$ & $p<0.001$ & \\
\hline & & $\mathrm{d} 21: 0.3 \mathrm{mg} / \mathrm{kg}, t_{(11)}=-8.31$ & $p<0.001$ & \\
\hline $5 H$ & Mann-Whitney test & $Z=-2.739$ & $p=0.004$ & l \\
\hline \multirow[t]{14}{*}{$6 B$} & Two-way repeated-measures ANOVA & $F_{(18,264)}=4.227$ & $p<0.001$ & Dunnett's multiple comparisons test (vs CaMKII-mCherry) \\
\hline & Interaction & $F_{(6,264)}=11.731$ & $p<0.001$ & Baseline: CaMKII-M3-mCherry+CN0 0.03 mg $/ \mathrm{kg}, p=0.989$; \\
\hline & Time effect & $F_{(3,44)}=24.086$ & $p<0.001$ & CaMKII-M3-mCherry+CN0 $0.1 \mathrm{mg} / \mathrm{kg}, p=1.000$; \\
\hline & Group effect & & & CaMKII-M3-mCherry+CNO 0.3 mg/kg, $p=0.394$ \\
\hline & & & & $1 \mathrm{~h}$ : CaMKII-M3-mCherry + CNO $0.03 \mathrm{mg} / \mathrm{kg}, p=0.882 ;$ \\
\hline & & & & CaMKII-M3-mCherry+CN0 $0.1 \mathrm{mg} / \mathrm{kg}, p=0.002$ \\
\hline & & & & CaMKIII-M3-mCherry+CN0 0.3 mg $/ \mathrm{kg}, p<0.001$ \\
\hline & & & & 2 h: CaMKII-M3-mCherry+CN0 $0.03 \mathrm{mg} / \mathrm{kg}, p=0.847$; \\
\hline & & & & CaMKII-M3-mCherry+CNO 0.1 mg/kg, $p<0.001$ \\
\hline & & & & CaMKIII-M3-mCherry+CN0 $0.3 \mathrm{mg} / \mathrm{kg}, p<0.001$ \\
\hline & & & & $3 \mathrm{~h}$ : CaMKII-M3-mCherry+CNO $0.03 \mathrm{mg} / \mathrm{kg}, p=0.978 ;$ \\
\hline & & & & CaMKII-M3-mCherry+CNO $0.1 \mathrm{mg} / \mathrm{kg}, p=0.002$ \\
\hline & & & & CaMKII-M3-mCherry+CNO 0.3 mg $/ \mathrm{kg}, p=0.001$ \\
\hline & & & & 4 h: CaMKII-M3-mCherry+ CNO 0.03 mg $/ \mathrm{kg}, p=1.000$; \\
\hline
\end{tabular}




\begin{tabular}{|c|c|c|c|c|}
\hline Figure. & Test & & & Post hoc comparison \\
\hline & & & & CaMKII-M3-mCherry+CN0 $0.1 \mathrm{mg} / \mathrm{kg}, p=0.008$; \\
\hline & & & & CaMKII-M3-mCherry+CNO $0.3 \mathrm{mg} / \mathrm{kg}, p=0.001$ \\
\hline & & & & 5 h: CaMKII-M3-mCherry+CN0 0.03 mg $/ \mathrm{kg}, p=0.995$; \\
\hline & & & & 0 CaMKII-M3-mCherry+CN0 $0.1 \mathrm{mg} / \mathrm{kg}, p=0.017$ \\
\hline & & & & 6 h: CaMKII-M3-mCherry+CN0 0.03 mg/kg, $p=0.999 ;$ \\
\hline & & & & CaMKII-M3-mCherry+CN0 $0.1 \mathrm{mg} / \mathrm{kg}, p=0.808$; \\
\hline & & & & CaMKII-M3-mCherry+CNO $0.3 \mathrm{mg} / \mathrm{kg}, p=0.509$ \\
\hline \multirow[t]{4}{*}{$6 C$} & Two-way repeated-measures ANOVA & $F_{(18,264)}=4.227$ & $p<0.001$ & Dunnett's multiple comparisons test (vs CaMKII-mCherry) \\
\hline & Interaction & $F_{(6,264)}=11.731$ & $p<0.001$ & CaMKII-M3-mCherry+CNO $0.03 \mathrm{mg} / \mathrm{kg}, p=0.847$; \\
\hline & Time effect & $F_{(3,44)}=24.086$ & $p<0.001$ & CaMKII-M3-mCherry+CNO $0.1 \mathrm{mg} / \mathrm{kg}, p<0.001$; \\
\hline & Group effect & & & CaMKII-M3-mCherry+CN0 0.3 mg/kg, $p<0.001$ \\
\hline \multirow[t]{3}{*}{$6 D$} & Paired-samples & Baseline: $0.03 \mathrm{mg} / \mathrm{kg}, t_{(11)}=-1.322$ & $p=0.213$ & \\
\hline & $t$ test & Baseline: $0.1 \mathrm{mg} / \mathrm{kg}, t_{(11)}=3955$ & $p=0.002$ & \\
\hline & & Baseline: $0.3 \mathrm{mg} / \mathrm{kg}, t_{(11)}=8.842$ & $p<0.001$ & \\
\hline $6 E$ & Mann-Whitney test & $Z=-2.887$ & $p=0.004$ & \\
\hline \multirow[t]{15}{*}{$7 C$} & Two-way repeated-measures ANOVA & $F_{(12,186)}=9.543$ & $p<0.001$ & Dunnett's multiple comparisons test (vs Control) \\
\hline & Interaction & $F_{(6,186)}=41.155$ & $p<0.001$ & Baseline: GFAP-M3 + CaMKII-mCherry + CN0 $0.3 \mathrm{mg} / \mathrm{kg}, p=1.000$; \\
\hline & Time effect & $F_{(2,31)}=97.073$ & $p<0.001$ & GFAP-M3 + CaMKII-M4-mCherry + CN0 $0.3 \mathrm{mg} / \mathrm{kg}, p=0.750$ \\
\hline & Group effect & & & 1 h: GFAP-M3 + CaMKII-mCherry + CNO $0.3 \mathrm{mg} / \mathrm{kg}, p=1.000$; \\
\hline & & & & GFAP-M3 + CaMKIII-M4-mCherry+CN0 0.3 mg/kg, $p<0.001$ \\
\hline & & & & $2 \mathrm{~h}$ : GFAP-M3+ CaMKII-mCherry+ CN0 $0.3 \mathrm{mg} / \mathrm{kg}, p=1.000$; \\
\hline & & & & GFAP-M3+CaMKII-M4-mCherry+CN0 $0.3 \mathrm{mg} / \mathrm{kg}, p<0.001$ \\
\hline & & & & $3 \mathrm{~h}:$ GFAP-M3 + CaMKIII-mCherry + CN0 $0.3 \mathrm{mg} / \mathrm{kg}, p=1.000$ \\
\hline & & & & GFAP-M3+CaMKII-M4-mCherry+CN0 $0.3 \mathrm{mg} / \mathrm{kg}, p<0.001$ \\
\hline & & & & 4 h: GFAP-M3+ CaMKIII-mCherry + CNO $0.3 \mathrm{mg} / \mathrm{kg}, p=1.000 ;$ \\
\hline & & & & GFAP-M3+CaMKII-M4-mCherry+CN0 $0.3 \mathrm{mg} / \mathrm{kg}, p<0.001$ \\
\hline & & & & 5 h: GFAP-M3 + CaMKII-mCherry + CN0 $0.3 \mathrm{mg} / \mathrm{kg}, p=1.000$ \\
\hline & & & & GFAP-M3+CaMKII-M4-mCherry+CN0 $0.3 \mathrm{mg} / \mathrm{kg}, p<0.001$ \\
\hline & & & & 6 h: GFAP-M3+ CaMKII-mCherry+ CN0 $0.3 \mathrm{mg} / \mathrm{kg}, p=1.000$; \\
\hline & & & & GFAP-M3 + CaMKII-M4-mCherry+CN0 $0.3 \mathrm{mg} / \mathrm{kg}, p=0.117$ \\
\hline \multirow[t]{4}{*}{$7 D$} & Two-way repeated-measures ANOVA & $F_{(12,186)}=9.543$ & $p<0.001$ & Dunnett's multiple comparisons test \\
\hline & Interaction & $F_{(6,186)}=41.155$ & $p<0.001$ & GFAP-M3+CaMKII-mCherry+CNO $0.3 \mathrm{mg} / \mathrm{kg}$, vs Control $p=1.000$ \\
\hline & Time effect & $F_{(2,31)}=97.073$ & $p<0.001$ & GFAP-M3+CaMKII-M4-mCherry+CN0 0.3 mg/kg, vs Control $p<0.001$ \\
\hline & Group effect & & & GFAP-M3+CaMKII-mCherry+CN0 $0.3 \mathrm{mg} / \mathrm{kg}$, vs GFAP-M3+CaMKII-M4-mCherry+CN0 $0.3 \mathrm{mg} / \mathrm{kg}, p<0.001$ \\
\hline \multirow[t]{6}{*}{$8 B$} & One-way ANOVA & $F_{(5,30)}=4.185$ & $p=0.007$ & LSD's multiple comparisons test \\
\hline & & & & Control vs DNP, $p=0.005$ \\
\hline & & & & DNP+GFAP-M4-mCherry vs DNP, $p=0.017$ \\
\hline & & & & DNP + GFAP-mCherry vs DNP+GFAP-M4-mCherry, $p=0.022$ \\
\hline & & & & GFAP-M3 + mCherry vs DNP, $p=0.484$ \\
\hline & & & & GFAP-mCherry vs GFAP-M $3+$ mCherry, $p=0.034$ \\
\hline \multirow[t]{6}{*}{$8 C$} & One-way ANOVA & $F_{(5,30)}=8.864$ & $p<0.001$ & LSD's multiple comparisons test \\
\hline & & & & Control vs DNP, $p<0.001$ \\
\hline & & & & DNP + GFAP-M4-mCherry vs DNP, $p=0.008$ \\
\hline & & & & DNP + GFAP-mCherry vs DNP+GFAP-M4-mCherry, $p=0.005$ \\
\hline & & & & GFAP-M3 + mCherry vs DNP, $p=0.937$ \\
\hline & & & & GFAP-mCherry vs GFAP-M3 + mCherry, $p<0.001$ \\
\hline \multirow[t]{4}{*}{ Extended Data 2-1 } & One-way ANOVA & $F_{(2,17)}=64.755$ & $p<0.001$ & Bonferroni multiple comparisons test \\
\hline & & & & DNP vs DNP + GFAP-M4-mCherry, $p<0.001$ \\
\hline & & & & DNP vs DNP + GFAP-M4-mCherry washout, $p=0.083$ \\
\hline & & & & DNP + GFAP-M4-mCherry vs DNP +GFAP-M4-mCherry washout, $p<0.001$ \\
\hline \multirow[t]{4}{*}{ Extended Data 3-1 } & One-way ANOVA & $F_{(2,16)}=110.537$ & $p<0.001$ & Bonferroni multiple comparisons test \\
\hline & & & & Control vs GFAP-M3-mCherry, $p<0.001$ \\
\hline & & & & Control vs GFAP-M3-mCherry washout, $p=1.000$ \\
\hline & & & & GFAP-M3-mCherry vs GFAP-M3-mCherry washout, $p<0.001$ \\
\hline
\end{tabular}

Statistical data with their respective post hoc comparison test for the behavioral immunohistochemical and ELISA data. In all cases, $p<0.05$ was used to indicate significance.

regarding DNP is the involvement of spinal astrocytes in pain modulation, little emphasis has been placed on supraspinal astrocytes. While the essential role of supraspinal astrocytes in providing structural and nutritional support for neurons has been elaborately expounded, their function in the modulation of DNP remains unclear.

Contrary to the traditional view that the cerebral cortex is not involved in pain perception, an extensive cortical network associated with pain processing has been revealed in recent years and is being increasingly recognized to play a major role in the representation and modulation of pain (Chen, 2018; Kragel et al., 2018). Since Tsubokawa and colleagues (Tsubokawa et al., 1991) initially reported long-term motor cortex (MCx) stimulation (MCS) as a potent therapy for neuropathic pain in 1991, many subsequent studies confirmed the reliability of MCS. Although the $\mathrm{MCx}$ is engaged in pain transmission, the exact 

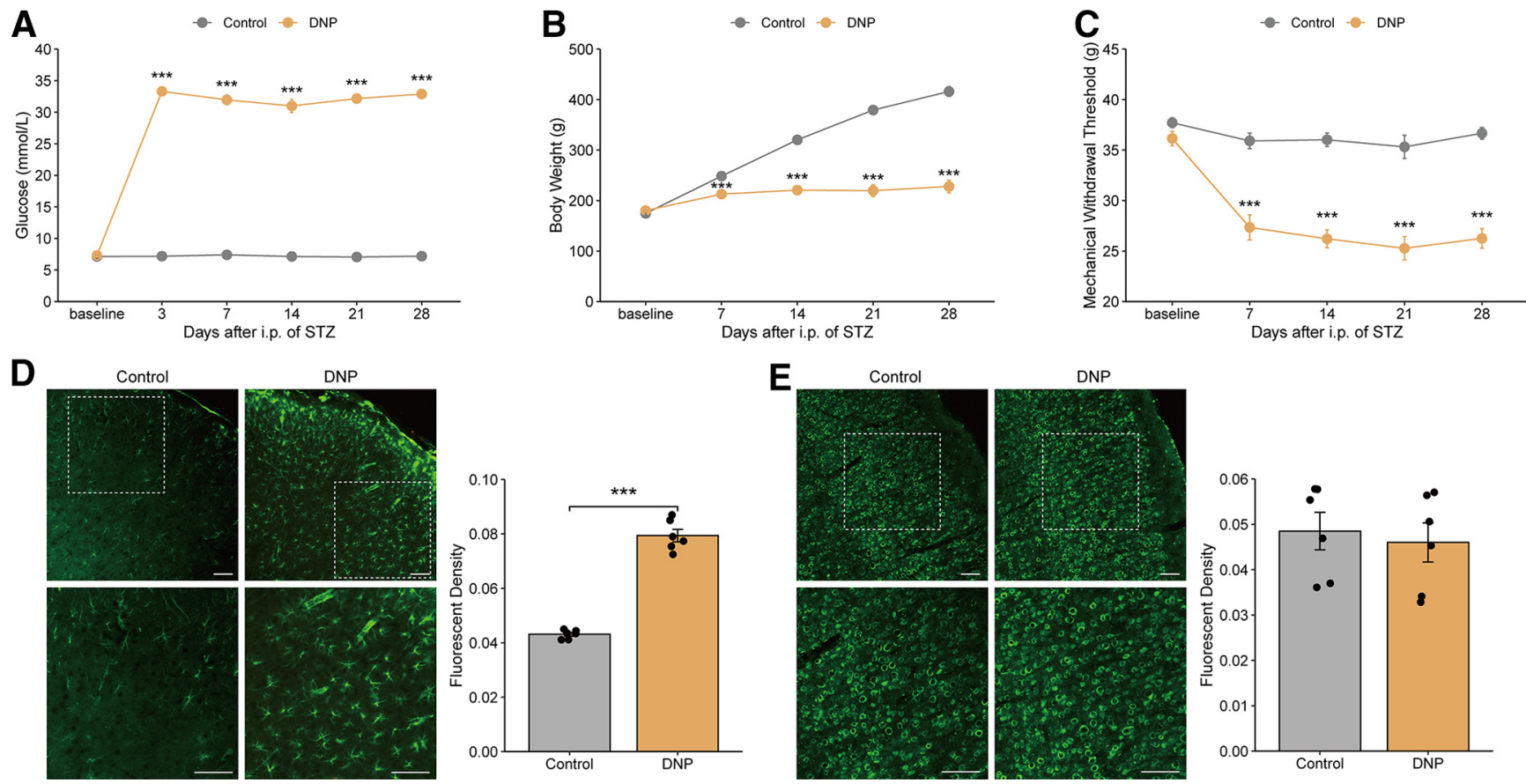

Figure 1. STZ-induced diabetic rats experienced mechanical allodynia accompanied by $M C x$ astrocyte activation. $(\boldsymbol{A})$ Blood glucose, $(\boldsymbol{B})$ body weight, and $(\boldsymbol{C})$ the mechanical withdrawal threshold levels were measured every $3 \mathrm{~d}$ or once weekly over 4 weeks after treatment with STZ. $* * * p<0.001$ versus the control group, $n=10$ or 11 rats/group, two-way repeated-measures ANOVA followed by unpaired $t$ test. (D) Immunofluorescence of GFAP or NeuN $(\boldsymbol{E})$ in the MCx region $31 \mathrm{~d}$ after the injection of vehicle or STZ. Bottom panels, High-magnification images of the top panels. Scale bars, $100 \mu \mathrm{m}$. Adjacent graph represents the quantification of fluorescence intensity. $* * * p<0.001$ versus the control group, $n=5$ or 6 rats/group, Student's $t$ test.

neuromechanisms related to MCS in neuropathic pain have not been fully clarified. A previous study detected significant activation of MCx astrocytes during the development of neuropathic pain in a rodent neonatal pain model (Sanada et al., 2014). However, the contribution of $\mathrm{MCx}$ astrocytes to neuropathic pain has not yet been determined. This transition of astrocytes to a reactive state can facilitate neuroinflammation, and the released inflammatory mediators can subserve neuron sensitization and amplify astrocyte activation, the latter of which is partly responsible for the progression of pain (Z. J. Zhang et al., 2017; Vanderwall and Milligan, 2019). Whether MCx glial cell hyperactivity and their interactions with local neurons (via the release of cytokines) contribute to the development and maintenance of DNP has not been studied.

To establish whether MCx glial cell hyperactivity and the glial cell release of cytokines that interact with local neurons contributes to the development and maintenance of DNP, we used the hM4Di designer receptor exclusively activated by designer drug (DREADD) to specifically inhibit astrocytes in the MCx region in DNP rats, and specific astrocyte activation was achieved by the hM3Dq DREADD in naive rats. The resultant changes in mechanical allodynia and the expression of $\mathrm{MCx}$ proinflammatory cytokines were measured. In detail, $\mathrm{MCx}$ astrocytes were inhibited in rats with DNP and activated in naive rats, the latter of which was performed either alone or in combination with the chemogenetic inhibition of MCx CAMKII neurons. Our results proved that MCx astrocytes are pivotal mediators of DNP and might shed light on treatment strategies for DNP.

\section{Materials and Methods}

Animals. Male Sprague Dawley rats (weighing 180-200 g) were obtained from the laboratory animal center of Fujian Medical University (license no. SCXK (Min) 2012-0001). All animals were housed in a controlled environment (temperature of $25 \pm 2^{\circ} \mathrm{C}$ with a $12 \mathrm{~h}$ light/dark cycle) with food and water available ad libitum. Before the experimental procedures, animals were randomly allocated into different groups. All experiments were performed in accordance with the guidelines of the Laboratory Animal Care and Use Committee of Fujian Medical University.

Induction of diabetic neuropathic pain and assessment. Rats were fasted overnight and then administered an intraperitoneal injection of $70 \mathrm{mg} / \mathrm{kg}$ fresh streptozotocin (STZ; Sigma Millipore) dissolved in $0.1 \mathrm{M}$ citrate buffer solution, pH 4.5 (Courteix et al., 1993). Animals in the control group received an equal volume of citrate buffer. The blood glucose levels of the tail vein were assessed using a One Touch Ultra Easy glucometer (Life Scan) $72 \mathrm{~h}$ after STZ injection. Rats with blood glucose levels $\geq 16.7 \mathrm{mmol} / \mathrm{L}$ were considered diabetic and included in the following study (Jiang et al., 2019). Diabetic rats with neuropathic pain were defined according to the ratio of the 21 st day mechanical withdrawal threshold (MWT) to the baseline MWT $<0.8$ as determined by a von Frey hair test (Fox et al., 1999).

Mechanical allodynia test. The mechanical allodynia of diabetic rats was assessed using a method reported by Mitrirattanakul et al. (2006) with minor modifications. Briefly, rats were individually placed in Plexiglas enclosures $(28 \times 22 \times 18 \mathrm{~cm})$ with wire mesh pads at the bottom and allowed to acclimate for at least $30 \mathrm{~min}$. Using a commercial electronic von Frey apparatus (model 2390, IITC Life Science), pressure was applied to the median plantar surface of the right hind paw by a von Frey fiber with a maximum pressure of $55 \mathrm{~g}$. A positive response was defined as an induced pain response (sudden withdrawal, shaking or licking of the hind paw) from the pressure of a filament, and the number on the liquid crystal display was then recorded. The test was performed at intervals of at least $5 \mathrm{~min}$ to eliminate interference from a previous stimulation. The test continued until three MWT readings were collected after the first change in response. The MWT for each subject was considered the mean of the three collected values. To minimize animal discomfort, mechanical nociceptive thresholds were tested in only one hind paw.

Drugs. AVV-GFAP-hM4Di(Gi)-mCherry $\left(10 \times 10^{12} \quad\right.$ V.G./ml $)$, AVV-GFAP-hM3Dq(Gq)-mCherry $\left(10 \times 10^{12}\right.$ V.G./ml), AVV-GFAPmCherry $\left(10 \times 10^{12}\right.$ V.G./ml), AVV-GFAP-hM3Di(Gq)-eGFP $(1.65 \times$ $10^{13}$ V.G./ml), HBAAV2/9-CaMKII-hM4Di-mCherry $\left(10 \times 10^{12}\right.$ V.G./ 
A

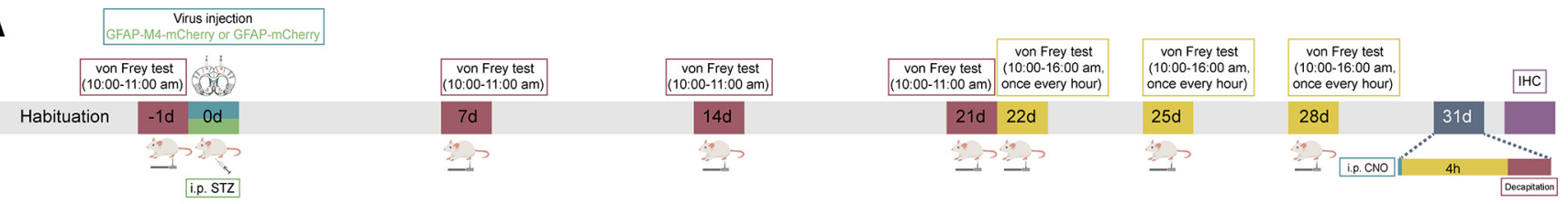

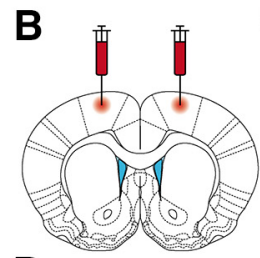

D

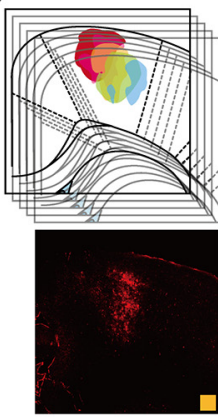

I
C
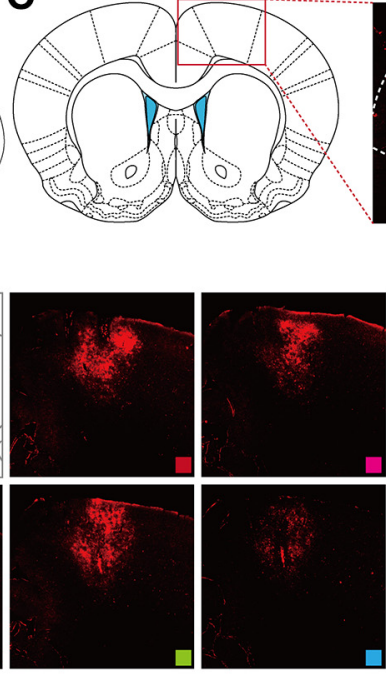

- DNP+GFAP-mCherry+CNO $(1.0 \mathrm{mg} / \mathrm{kg})$

- DNP+GFAP-M4-mCherry+CNO $(0.1 \mathrm{mg} / \mathrm{kg})$

- DNP+GFAP-M4-mCherry+CNO $(0.3 \mathrm{mg} / \mathrm{kg})$

- DNP+GFAP-M4-mCherry+CNO $(1.0 \mathrm{mg} / \mathrm{kg})$

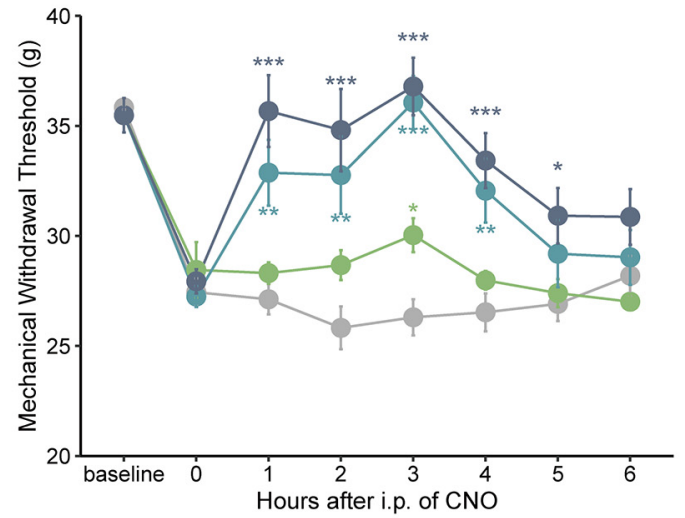

K

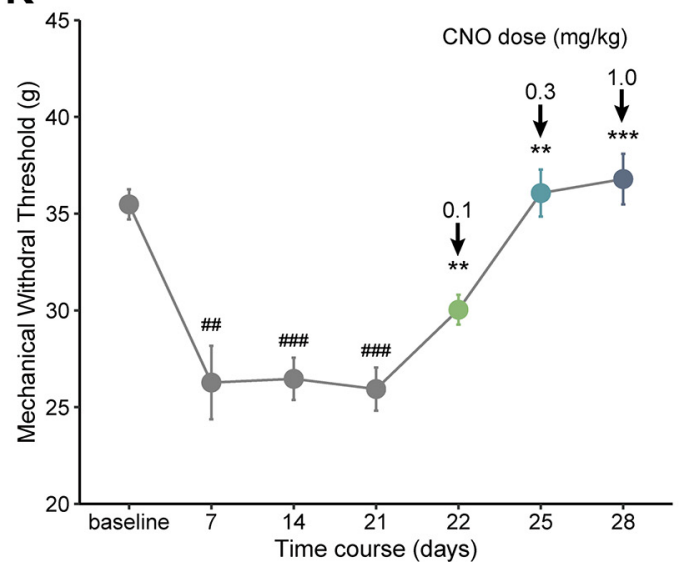

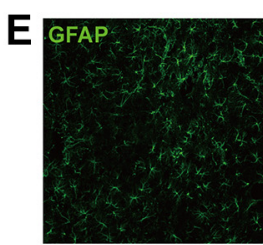

$\mathbf{F}$ NeuN

H
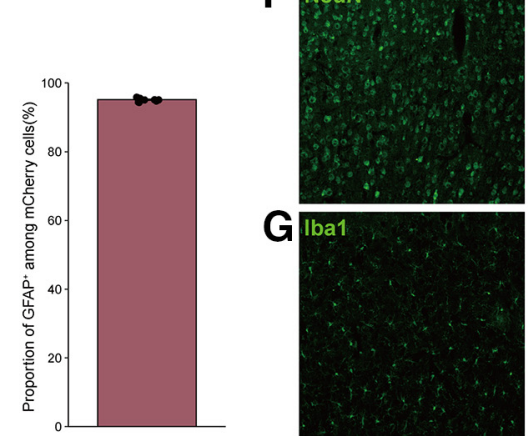

G |ba1
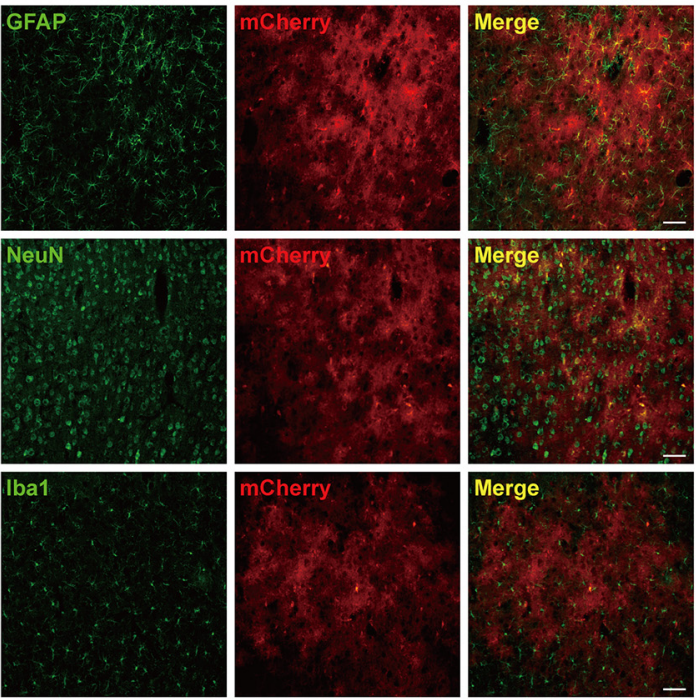

Merge

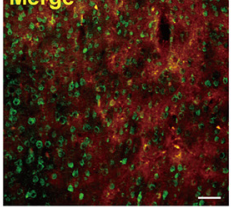

Merge

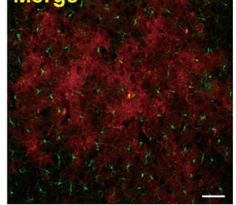

J

$D N P+G F A P-m C h e r r y+C N O(1.0 \mathrm{mg} / \mathrm{kg})$

DNP+GFAP-M4-mCherry+CNO $(0.1 \mathrm{mg} / \mathrm{kg})$

DNP+GFAP-M4-mCherry+CNO $(0.3 \mathrm{mg} / \mathrm{kg})$

DNP+GFAP-M4-mCherry+CNO $(1.0 \mathrm{mg} / \mathrm{kg})$

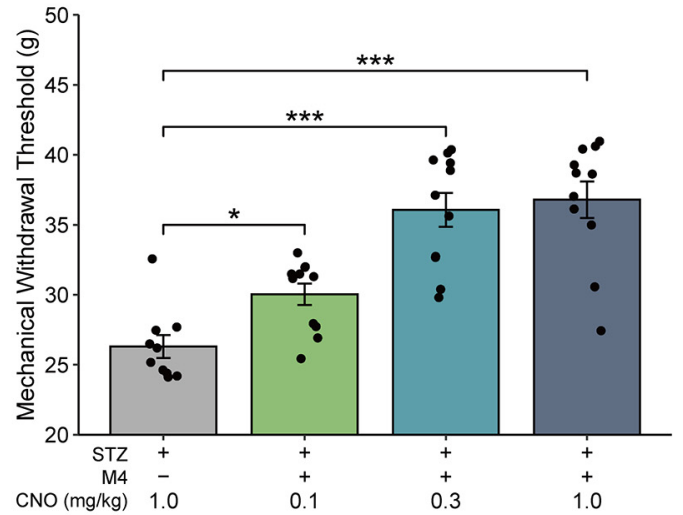

L

DNP+GFAP-mChery DNP+GFAP-M4-mChery
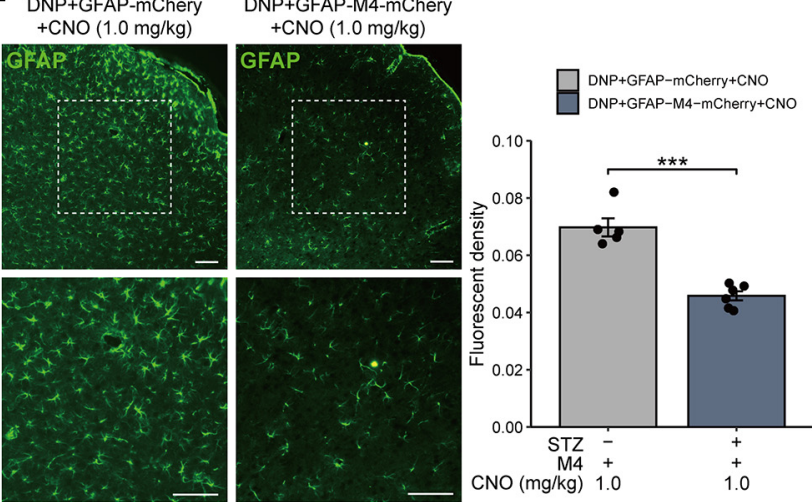

Figure 2. Selective inhibition of astrocytes via GFAP-M4-mCherry in the MCx reverses mechanical allodynia in rats with DNP. $\boldsymbol{A}$, Timeline of experiments. $\boldsymbol{B}$, The GFAP-M4-mCherry virus was injected into the bilateral $M C x$ region of rats. C, The virus construct contained a red mCherry tag, and red fluorescence was used to identify the expression of GFAP-M4 in the MCx. D, Series brain slices of GFAP-M4-mCherry expression in MCX. E, Green represents astrocyte marker (GFAP) labeling. Red represents the virus tag. Red-green merged image represents virus colocalization with GFAP. Scale bars, $100 \mu \mathrm{m}$. $\boldsymbol{F}$, Green represents neuronal marker (NeuN) labeling. Red represents the virus tag. Red-green merged image confirms that the virus merged exclusively with GFAP and did not colocalize with NeuN. Scale bars, $100 \mu \mathrm{m}$. G, Green represents microglial marker (Iba1) labeling. Red represents the viral tag. Merged red-green image confirms that the virus 
ml), HBAAV2/9-CaMKII- hM3Dq-mCherry $\left(10 \times 10^{12}\right.$ V.G./ml $)$, and HBAAV2/9-CaMKII-mCherry $\left(10 \times 10^{12}\right.$ V.G./ml $)$ were purchased from Shanghai Hanbio Biotechnology. Clozapine-N-oxide (CNO) was purchased from LKT Laboratories (C4759) and was dissolved in sterile saline.

Stereotactic virus injection. Rats were anesthetized with isoflurane (Shenzhen RWD Life Science) and placed in an automated stereotactic aperture (RWD68001, Shenzhen RWD). A small incision was made in the head to expose the skull, and a small craniotomy was performed. Rats were directed bilaterally in the $\mathrm{MCx}$ region (coordinates: $\mathrm{AP}=$ $\pm 1.7 \mathrm{~mm}$; $\mathrm{ML}= \pm 1.5 \mathrm{~mm}$; DV $=-1.4 \mathrm{~mm}$; bregma zero) using a $1 \mu \mathrm{l}$ glass microsyringe for microinjection. The injection volume $(500 \mathrm{nl} /$ hemisphere) and rate (60 nl/minute) were controlled by an injection pump (RWD 68606, Shenzhen RWD Life Science). After the injection, the microsyringe was left in place for at least $10 \mathrm{~min}$ to prevent virus from spreading through the injector track, and the injector was then slowly removed. The incision was closed with disposable sterile sutures and needles; for postoperative care, rats were administered an intraperitoneal injection of benzylpenicillin (Henan Xinxiang Hua Xing Pharmaceutical Factory) dissolved in saline. The conditions of the rats were carefully observed until they recovered, and the rats were then placed in their original cages. Rats were injected intraperitoneally with $0.03-1.0 \mathrm{mg} / \mathrm{kg}$ CNO before the behavioral experiment.

Immunohistochemical staining. The brains were sectioned at $20 \mu \mathrm{m}$ on a freezing microtome after perfusion and fixation in 4\% PFA. The sections were blocked with $10 \%$ donkey serum and then incubated with the appropriate primary antibody (anti-GFAP, 1:500 dilution, 3670S, Cell Signaling Technology; anti-NeuN, 1:500 dilution, ab104224, Abcam; anti-Iba1, 1:500 dilution, 019-19741, Wako Chemicals; anti-cFos, 1:500 dilution, ABE457, Merck KGaA; anti-CaMKII, 1:200 dilution, ab2260, Abcam; and anti-GABA, 1:200 dilution, a2052, Sigma Millipore) and secondary antibody (EarthOx) successively. All images were obtained by fluorescence microscopy (Leica DM2000, Leica Microsystems) with Leica Application Suite-X software (Leica Microsystems, version 3.4.1). The fluorescence density was analyzed with Image-Pro Plus software (Media Cybernetics, version 6.0).

ELISA. Rats were deeply anesthetized with pentobarbital sodium $(30 \mathrm{mg} / \mathrm{kg}$ ) and then killed by decapitation. Then, the MCx tissues were harvested for homogenization and stored at $-80^{\circ} \mathrm{C}$. Standard ELISA procedures were performed in accordance with the manufacturer's instructions to determine TNF- $\alpha$ and IL- $1 \beta$ expression. The concentrations of TNF- $\alpha$ and IL- $1 \beta$ are presented as $\mathrm{pg} / \mathrm{mg}$ protein in the tissue homogenate.

Data analysis. Analysis was performed using IBM SPSS Statistics software (version 20.0). All data are presented as the mean \pm SEM and were analyzed statistically by Student's $t$ test, the paired-samples $t$ test, the Wilcoxon signed-rank test, the Mann-Whitney test, one-way ANOVA or two-way repeated-measures ANOVA according to the

$\leftarrow$

merged exclusively with GFAP and did not colocalize with microglia. Scale bars, $100 \mu \mathrm{m} . \boldsymbol{H}$, GFAP-M4-mCherry was expressed in $>95 \%$ of MCX astrocytes (2709 of 2817 cells from 10 rats). I, Mechanical pain hypersensitivity of rats expressing GFAP-M4-mCherry or GFAPmCherry before CNO injection $(0 \mathrm{~h})$ and over a $6 \mathrm{~h}$ time course after CNO treatment. $\# p<0.001$ versus baseline; $* p<0.05, * * p<0.01, * * * p<0.001$ versus the DNP + GFAP-mCherry group, $n=10$ or 11 rats/group, two-way repeated-measures ANOVA followed by Dunnett's multiple comparisons test. J, Maximal possible effects of selective astrocyte inhibition in the MCX at $3 \mathrm{~h}$ after CNO injection on STZ-induced mechanical allodynia. $* p<0.05$, $* * * p<0.001$ versus the DNP+GFAP-mCherry group, $n=10$ or 11 rats/ group, two-way repeated-measures ANOVA followed by Dunnett's multiple comparisons test. $\boldsymbol{K}$, The effects of different doses of CNO on mechanical allodynia after STZ injection were evaluated $3 \mathrm{~h}$ after each CNO administration in GFAP-M4-mCherry-expressing rats with DNP. $\# p<0.01, \# \# p 0.001$ versus baseline; $* * p<0.01$, $* * * p<0.001$ versus day 21 before CNO treatment, $n=10$ or 11 rats/group, paired-samples $t$ test or Wilcoxon signed-rank test. $L$, The GFAP-M4-mCherry-induced selective inhibition of astrocytes in the MCx region of rats with DNP attenuates the GFAP fluorescence intensity. Scale bars, $100 \mu \mathrm{m}$. Adjacent graph represents the quantification of fluorescence intensity. $* * * p<0.001$ versus the DNP + GFAP-mCherry group, $n=5$ or 6 rats/group, Student's $t$ test. experimental protocol, followed by the Games-Howell, pairwise, least significant difference (LSD) or Dunnett's T3 post hoc test, as appropriate. Probability values $<0.05$ were considered statistically significant. The statistical analysis details are shown in Table 1.

\section{Results}

\section{MCx astrocytes were activated in rats with DNP}

Following intraperitoneal injection of STZ, the concentration of blood glucose was significantly increased within $3 \mathrm{~d}$ and maintained throughout the experiment (Fig. 1A). In addition, all STZtreated rats suffered from the typical clinical symptoms of diabetes and exhibited polydipsia, polyuria, polyphagia, and diarrhea. Despite significant increases in food and water intake, the body weights of the STZ-treated rats were profoundly reduced (Fig. $1 B)$. Mechanical hypersensitivity was consistently present in diabetic rats at least 4 weeks after STZ injection, evidenced by reductions in their MWTs compared with their basal values and the values of the control rats. Then, the MWT reached a minimum at week 3 and continued thereafter (Fig. 1C). Based on these characteristics, STZ injection can induce persistent diabetes in rats.

To determine whether astrocytes are activated in the $\mathrm{MCx}$ regions of diabetic rats that display tactile allodynia, we immunostained MCX samples from rats taken $31 \mathrm{~d}$ after injection of STZ. GFAP expression was significantly increased at $31 \mathrm{~d}$ in rats with DNP (Fig. 1D) but was not changed in control animals. In contrast, the immunostaining level of $\mathrm{NeuN}$, a marker of neurons, in the $\mathrm{MCx}$ region of rats with DNP was not different from that in control rats (Fig. 1E). The significantly increased expression of GFAP in the MCx after the development of DNP suggests a potential role of MCx astrocytes in the pathogenesis of DNP.

\section{Chemogenetic inhibition of $\mathrm{MCx}$ astrocytes reverses mechanical allodynia in rats with DNP}

To investigate the regulation of MCx astrocytes on mechanical allodynia in rats with DNP, we applied chemogenetic methods to manipulate $\mathrm{MCx}$ astrocytes. First, rats were intraperitoneally injected with STZ to induce DNP. Then, we bilaterally injected the GFAP-M4-mCherry virus into the $\mathrm{MCx}$ region to inhibit MCx astrocytic activity. Approximately 4 weeks after virus transfection, red fluorescence was observed to be restricted to the $M C x$ region (Fig. $2 B-D$ ). Within the virally transduced region, GFAP-M4-mCherry expression was limited to the astrocytes (Fig. $2 E$ ), with high penetrance $(>95 \%$ of the GFAP cells expressed mCherry) (Fig. $2 H$ ), and the noncolocalization of mCherry and NeuN or Ibal demonstrated the specific expression of the GFAP-M4-mCherry-labeled virus in astrocytes instead of neurons or microglia (Fig. $2 F, G$ ).

Three weeks after the GFAP-M4-mCherry virus injection, different doses of $\mathrm{CNO}(0.3-1.0 \mathrm{mg} / \mathrm{kg}$, i.p.) were administered from days 22 to 28 after STZ injection, and pain was assessed during a $6 \mathrm{~h}$ time course after each administration (Fig. $2 A$ ). In the DNP+GFAP-M4-mCherry group, STZ-induced mechanical allodynia was reversed at $1 \mathrm{~h}$, peaked at $3 \mathrm{~h}$, and remained positive for $5 \mathrm{~h}$ after CNO treatment (Fig. 2I). To test whether these effects were selectively mediated by the $\mathrm{CNO}$-induced activation of GFAP-M4-mCherry, we administered CNO to rats in which GFAP-M4-mCherry expression was absent, and we observed no relief in mechanical allodynia. Compared with that in the $\mathrm{DNP}+\mathrm{GFAP}$-mCherry group, the MWT of DNP rats expressing GFAP-M4-mCherry was markedly increased by CNO (Fig. 2J). Moreover, different doses of $\mathrm{CNO}$ could alleviate mechanical allodynia in a dose-dependent manner (Fig. 2K). 
A
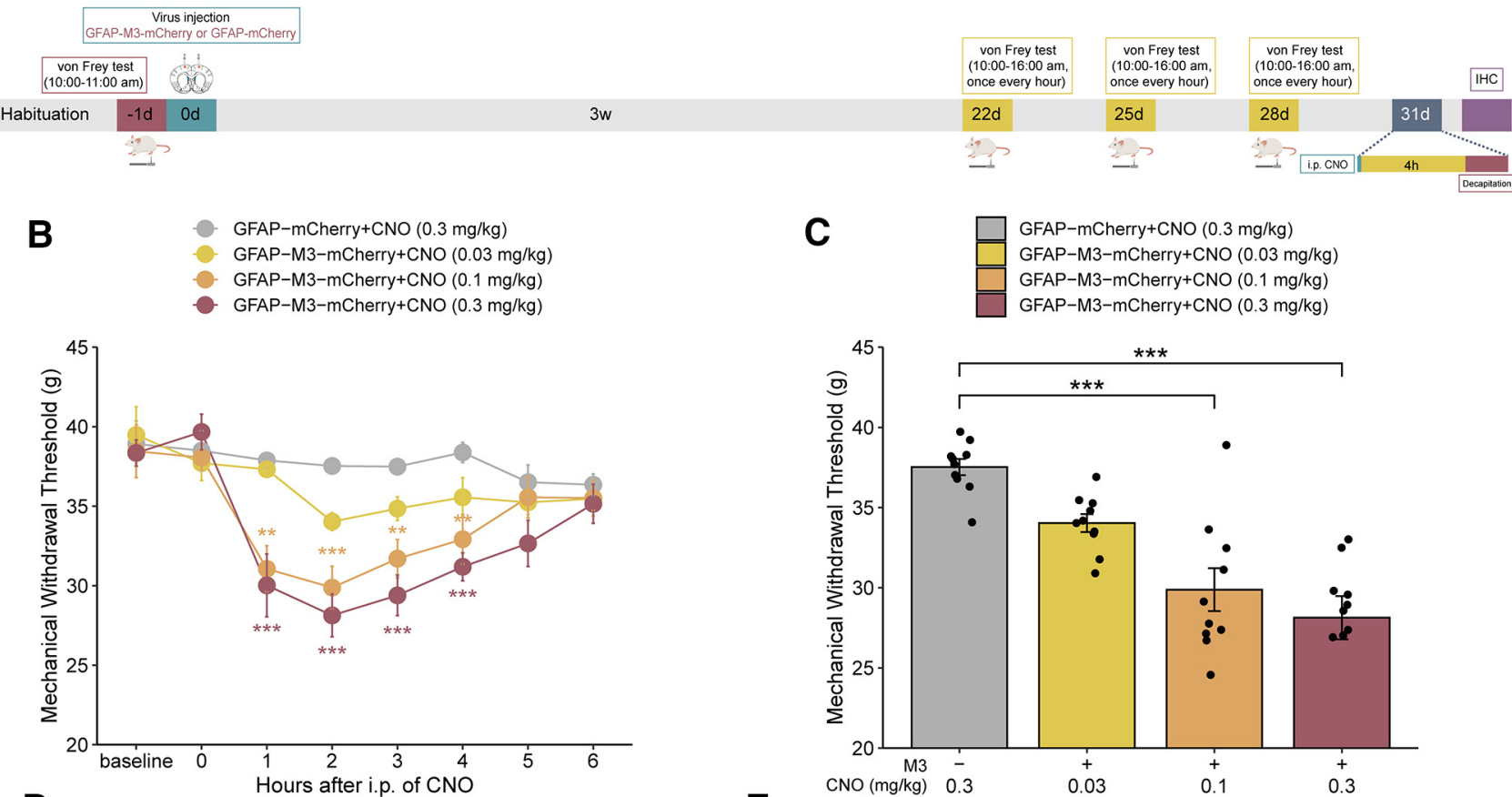

D

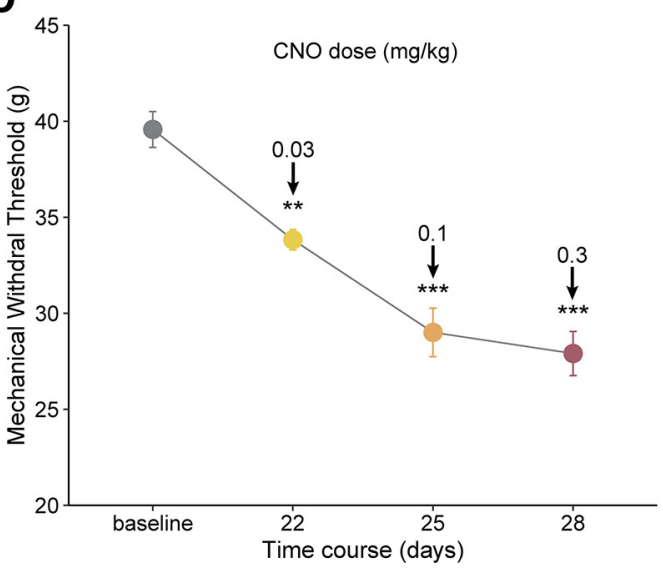

C

C GFAP-mCherry+CNO $(0.3 \mathrm{mg} / \mathrm{kg})$

GFAP-M3-mCherry+CNO $(0.03 \mathrm{mg} / \mathrm{kg})$

GFAP-M3-mCherry+CNO $(0.1 \mathrm{mg} / \mathrm{kg})$

GFAP-M3-mCherry+CNO $(0.3 \mathrm{mg} / \mathrm{kg})$

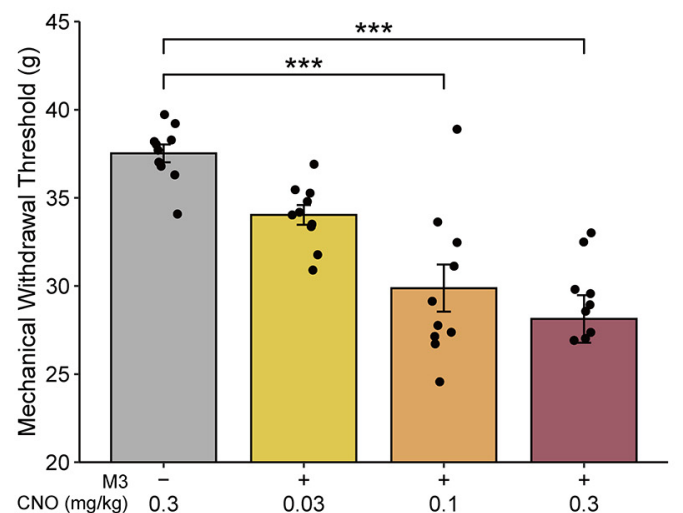

E $\mathrm{CNO}(\mathrm{mg} / \mathrm{kg}) \quad 0.3$
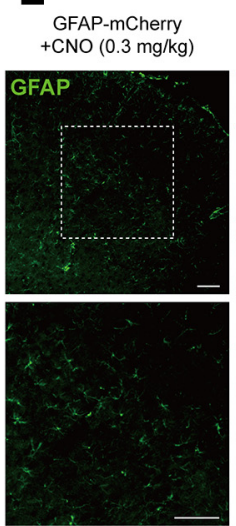

GFAP-M3-mCherry + CNO $(0.3 \mathrm{mg} / \mathrm{kg})$

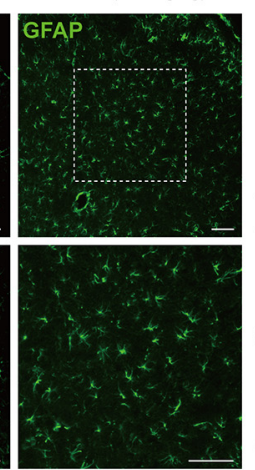

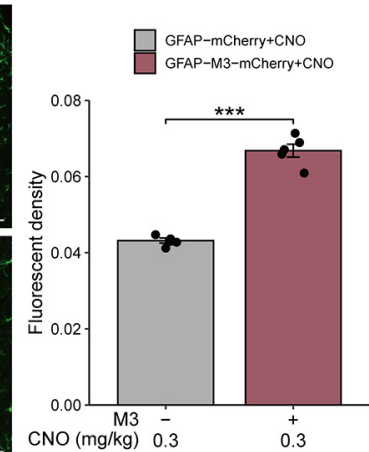

Figure 3. Selective activation of astrocytes in the MCx via GFAP-M3-mCherry induces mechanical allodynia in naive rats. $\boldsymbol{A}$, Timeline of the experiments. $\boldsymbol{B}$, Mechanical pain hypersensitivities of rats expressing GFAP-M3-mCherry or GFAP-mCherry before the CNO injection $(0 \mathrm{~h})$ and over a $6 \mathrm{~h}$ time course after CNO treatment. ${ }^{* *} p<0.01$, ${ }^{* * *} p<0.001$ versus the GFAP-mCherry group, $n=10$ rats/group, two-way repeated-measures ANOVA followed by Dunnett's multiple comparisons test. C, Maximal possible effects of the selective activation of astrocytes in the MCX at $2 \mathrm{~h}$ after CNO injection on mechanical allodynia in naive rats. ${ }^{* * *} p<0.001$ versus the GFAP-mCherry group, $n=10$ rats/group, two-way repeated-measures ANOVA followed by Dunnett's multiple comparisons test. $\boldsymbol{D}$, The effects of different doses of CNO on mechanical allodynia after virus injection were evaluated $2 \mathrm{~h}$ after each CNO administration in rats expressing GFAP-M3mCherry. ${ }^{* *} p<0.01,{ }^{* * *} p<0.001$ versus baseline, $n=10$ rats/group, paired-samples $t$ test. $\boldsymbol{E}$, Selective activation of astrocytes in the MCx of naive rats via GFAP-M3-mCherry significantly increased the GFAP fluorescence intensity. Scale bars, $100 \mu \mathrm{m}$. Adjacent graph represents the quantification of fluorescence intensity. ${ }^{* * *} p<0.001$ versus the GFAP-mCherry group, $n=5$ rats/ group, Student's $t$ test.

To determine the effects of GFAP-M4-mCherry receptor activation by $\mathrm{CNO}$ on astrocyte activity, we examined the expression of GFAP in the MCx region after staining with a GFAP antibody. As expected, CNO treatment significantly attenuated the GFAP fluorescence in rats with DNP-expressing GFAP-M4-mCherry compared with rats with DNP-expressing GFAP-mCherry (Fig. $2 L$ ). What's more, photomicrographs illustrating after $\mathrm{CNO}$ washout the GFAP signal in DNP+GFAP-M4-mCherry group had no difference compared with the DNP group (Extended Data Fig. 2-1).

\section{Chemogenetic activation of MCx astrocytes induces} mechanical allodynia in naive rats

To further investigate whether the activation of MCx astrocytes is sufficient to cause mechanical allodynia, we further activated $\mathrm{MCx}$ astrocytes with the GFAP-M3-mCherry virus. To activate
MCx astrocytes, naive rats were bilaterally injected with GFAPM3-mCherry in the MCx region. Three weeks after the virus injection, $\mathrm{CNO}$ was administered at different doses (0.03$0.3 \mathrm{mg} / \mathrm{kg}$, i.p.) from days 22 to 28 , and pain was assessed during a $6 \mathrm{~h}$ time course after each administration (Fig. $3 A$ ). In the GFAP-M3-mCherry group, mechanical allodynia was induced gradually, peaked at $2 \mathrm{~h}$ after $\mathrm{CNO}$ administration, and then returned to normal $6 \mathrm{~h}$ after $\mathrm{CNO}$ treatment. However, no significant change in MWT was found in the GFAP-mCherry group (Fig. 3B). Compared with that in the GFAP-mCherry group, CNO caused the MWT to decrease significantly in the GFAP-M3 group (Fig. 3C), and different doses of $\mathrm{CNO}$ could lower mechanical allodynia to varying degrees (Fig. 3D).

To determine the effects of GFAP-M3-mCherry receptor activation by $\mathrm{CNO}$ on astrocyte activity, the expression of GFAP in 

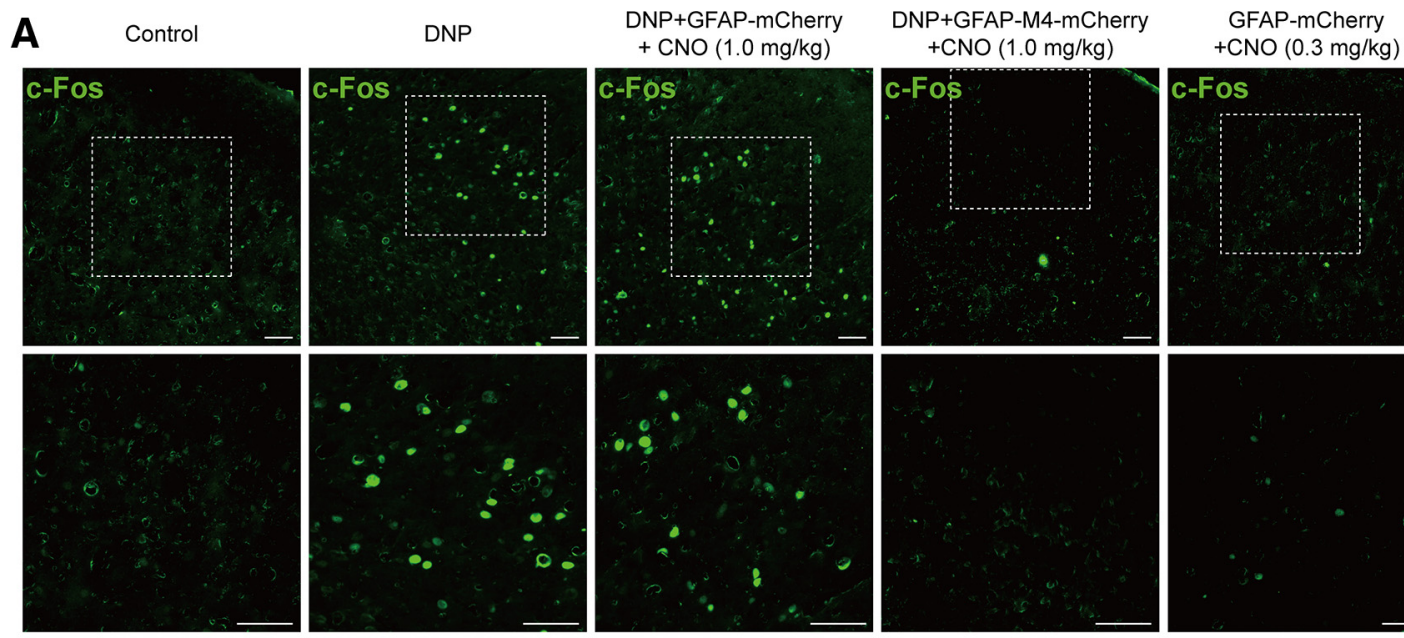

GFAP-M3-mCherry
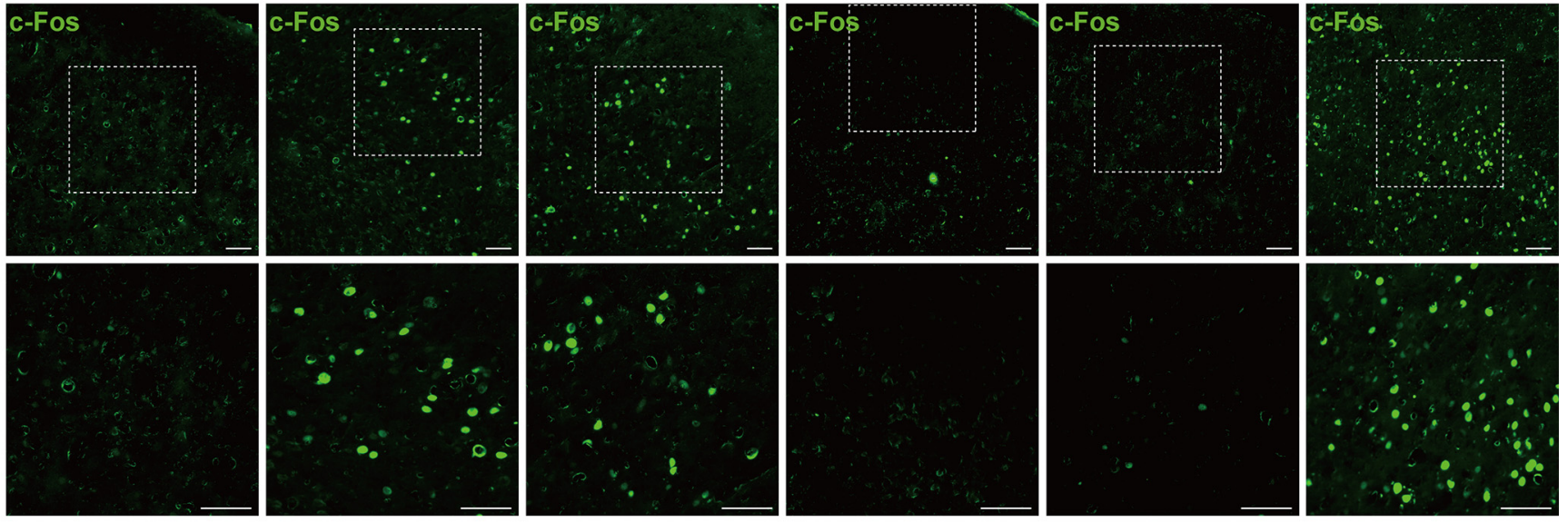

$\mathbf{B} \quad \square \underset{\text { DNP }}{\text { Control }} \square \begin{aligned} & \text { DNP+GFAP-mCherry+CNO } \\ & \text { DNP+GFAP-M4-mCherry+CNO }\end{aligned} \square \begin{aligned} & \text { GFAP-mCherry+CNO } \\ & \text { GFAP-M3-mCherry+CNO }\end{aligned}$
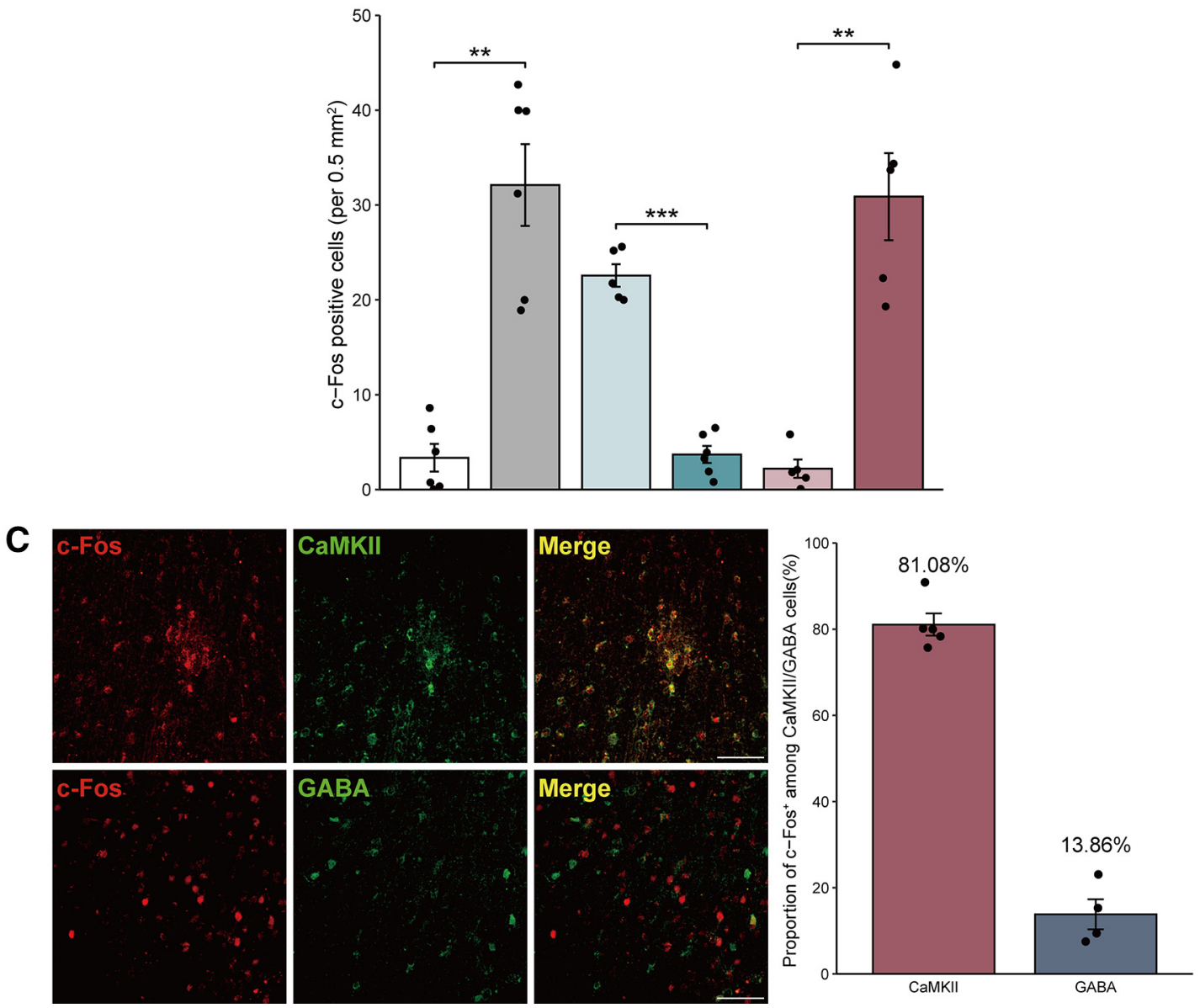

Figure 4. MCX astrocytes affect the activity of MCX excitatory neurons. $A$, The effects of GFAP-M4-mCherry or GFAP-M3-mCherry on c-Fos in rats with or without STZ injection. Scale bars, $100 \mu \mathrm{m} . \boldsymbol{B}$, Immunohistochemistry quantification of c-Fos-positive cells. ${ }^{* *} p<0.01$, ${ }^{* * *} p<0.001$ versus the control or GFAP-mCherry group, $n=5$ or 6 rats/group, separate one-way ANOVA followed by the Games-Howell multiple comparisons test. C, Photomicrographs illustrating the double immunofluorescence staining of CaMKII (green) or GABA (green) with c-Fos (red) in the $M C X$ of rats with DNP. The vast majority of c-Fos-positive cells were also positive for CaMKIl, and only a few c-Fos-positive cells were also positive for GABA. Adjacent graph represents the proportion of c-Fos-positive cells that were also positive for CaMKII or GABA cells. Scale bars, $100 \mu \mathrm{m} . n=5$ or 6 rats/group.

the MCx region was examined after staining with a GFAP antibody. The immunofluorescence staining of MCx indicated that GFAP expression was significantly enhanced in rats injected with GFAP-M3-mCherry compared with those injected with GFAP-mCherry (Fig. 3E). What's more, photomicrographs illustrating after CNO washout the GFAP signal in GFAP-M3-
mCherry group had no difference compared with the control group (Extended Data Fig. 3-1).

MCx excitatory neurons are regulated by local astrocytes Astrocytes are in close contact with neuronal synapses, regulate the excitation of neurons (Scholz and Woolf, 2007), and promote 
A

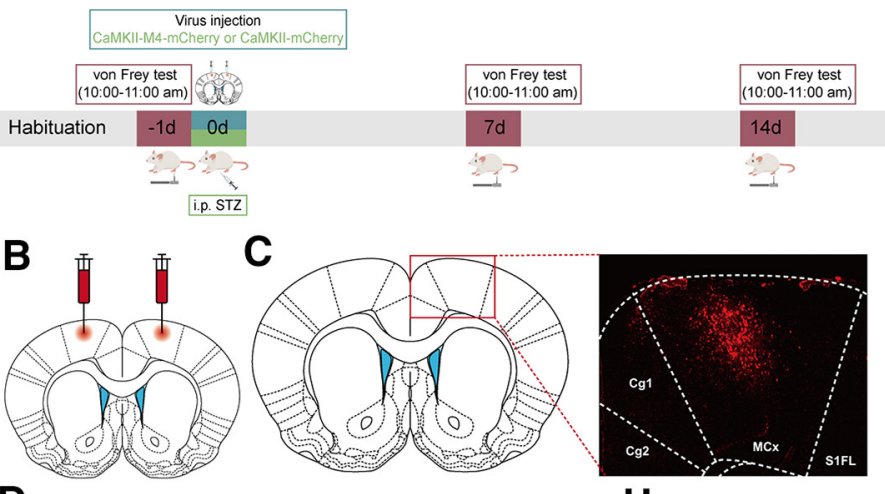

D

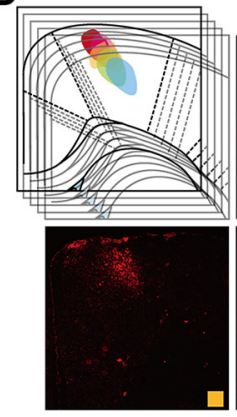

I

- DNP+CaMKII-mCherry+CNO $(0.3 \mathrm{mg} / \mathrm{kg})$

- DNP+CaMKII-M4-mCherry+CNO $(0.03 \mathrm{mg} / \mathrm{kg})$

- DNP+CaMKII-M4-mCherry+CNO $(0.1 \mathrm{mg} / \mathrm{kg})$

- DNP+CaMKII-M4-mCherry+CNO $(0.3 \mathrm{mg} / \mathrm{kg})$
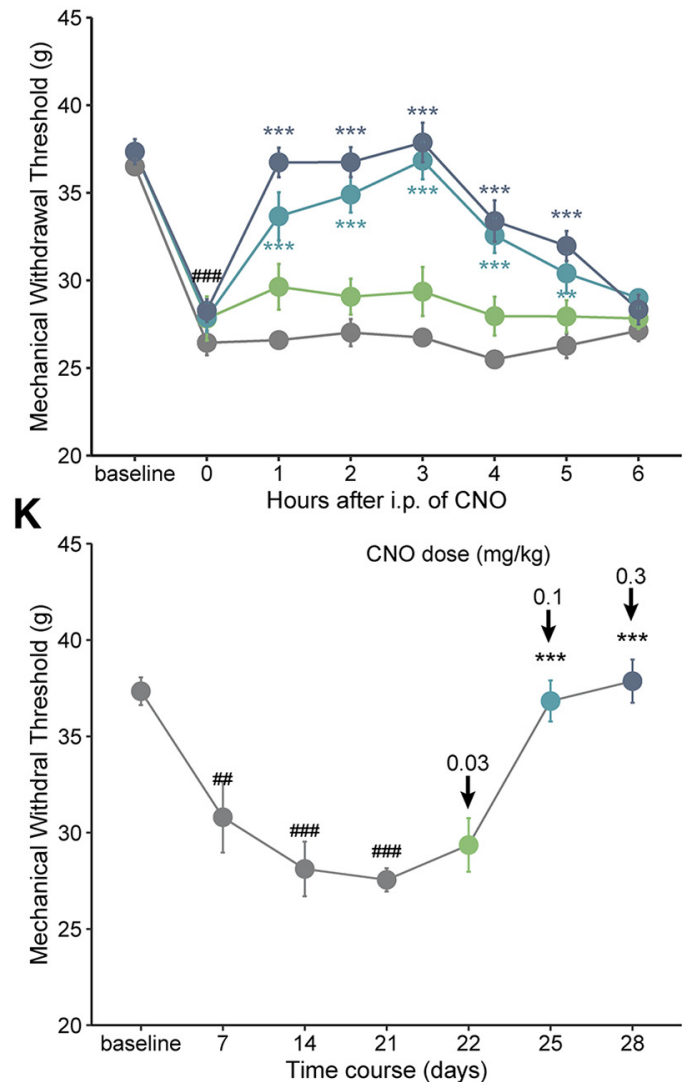
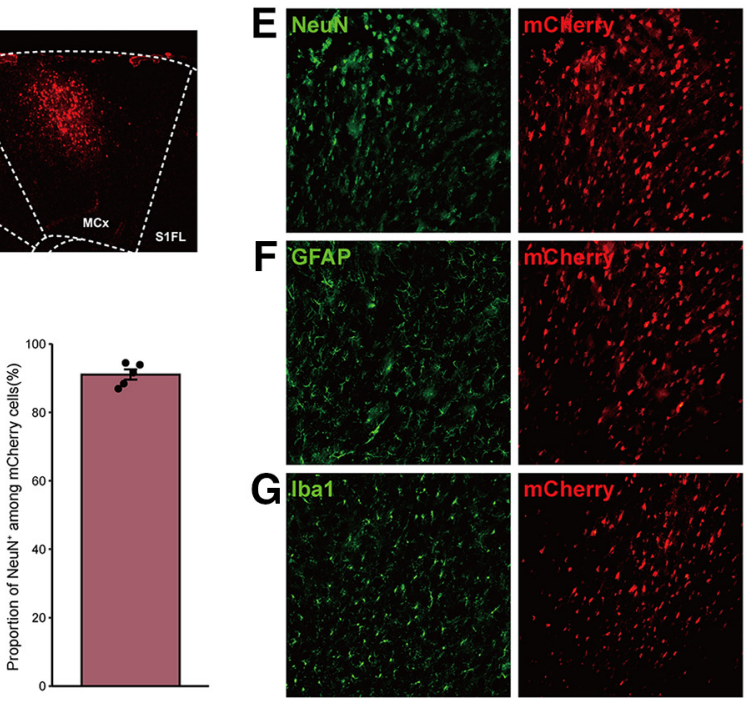

$\mathbf{J}$
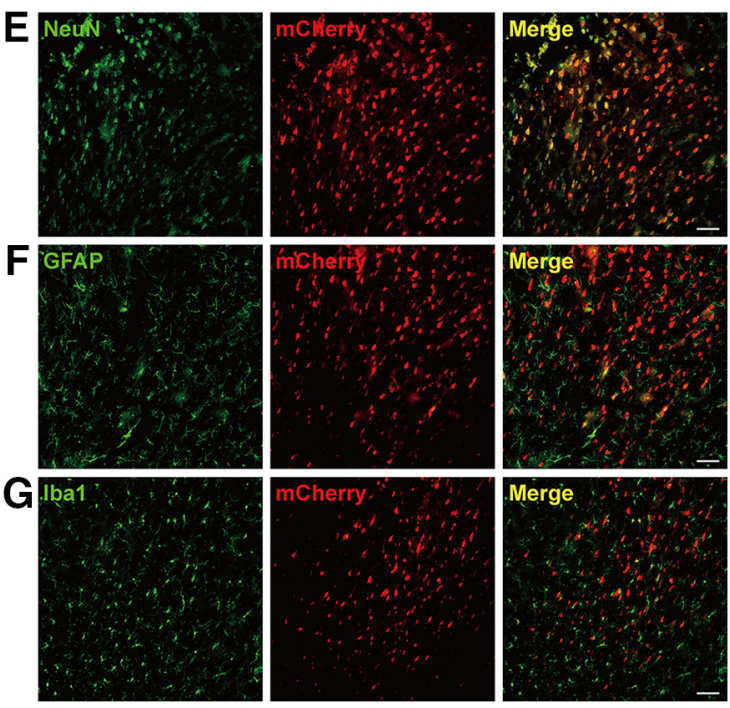

DNP+CaMKII-mCherry+CNO $(0.3 \mathrm{mg} / \mathrm{kg})$

$\mathrm{DNP}+\mathrm{CaMKII}-\mathrm{M} 4-\mathrm{mCherry}+\mathrm{CNO}(0.03 \mathrm{mg} / \mathrm{kg})$ $\mathrm{DNP}+\mathrm{CaMKII}-\mathrm{M} 4-\mathrm{mCherry}+\mathrm{CNO}(0.1 \mathrm{mg} / \mathrm{kg})$ $\mathrm{DNP}+\mathrm{CaMKII}-\mathrm{M} 4-\mathrm{mCherry}+\mathrm{CNO}(0.3 \mathrm{mg} / \mathrm{kg})$

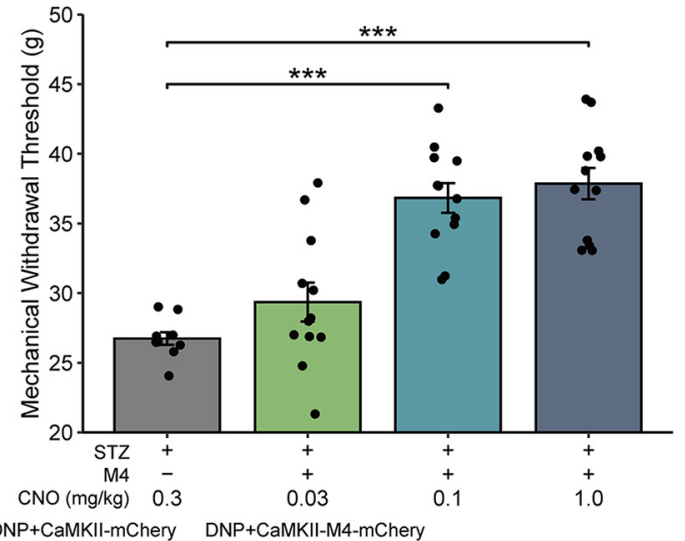

L DNP+CaMKII-mChery DNP+CaMKII-M4-mChery $+\mathrm{CNO}(0.3 \mathrm{mg} / \mathrm{kg})$
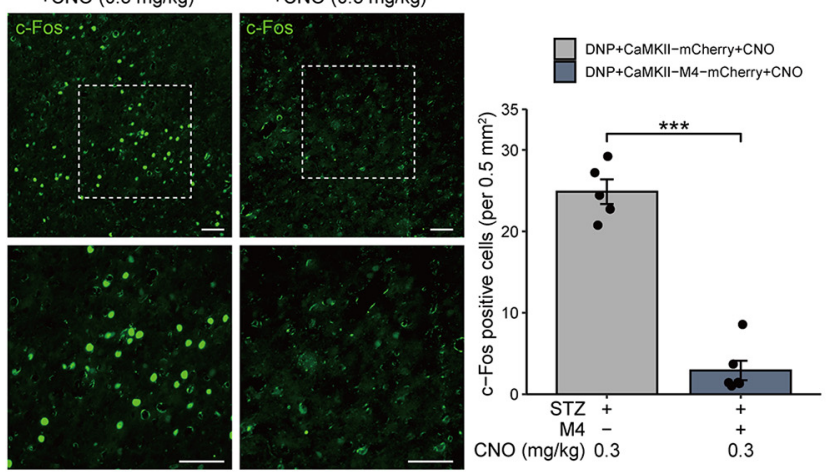

Figure 5. Selective inhibition of MCX excitatory neurons via CaMKII-M4-mCherry reverses mechanical allodynia in rats with DNP. $\boldsymbol{A}$, Timeline of the experiments. $\boldsymbol{B}$, The CaMKII-M4-mCherry virus was injected bilaterally into the rat MCx. C, The virus construct contained a red mCherry tag, and red fluorescence was used to identify the expression of CaMKII-M4 in the MCx. $\mathbf{D}$, The CaMKII-M4-mCherry virus was expressed in different parts of MCX. $\boldsymbol{E}$, Green represents neuronal marker (NeuN) labeling. Red represents the virus tag. Red-green merged image represents virus colocalization with NeuN. Scale bars, $100 \mu \mathrm{m}$. $\boldsymbol{F}$, Green represents astrocyte marker (GFAP) labeling. Red represents the virus tag. Red-green merged image confirms that the virus rarely colocalized with GFAP. Scale bars, $100 \mu \mathrm{m}$. G, Green represents microglial marker (Iba1) labeling. Red represents the virus tag. Merged red-green image confirms that the virus merged exclusively 
the maintenance of pain by releasing inflammatory factors (Guo et al., 2007). Therefore, we detected the expression of c-Fos in the MCx to further investigate whether specific modulation of MCx astrocytes affects the activation of MCX neurons in rats with DNP. c-Fos was hardly found in the $\mathrm{MCx}$ region of the control group, while c-Fos expression in the DNP group was significantly increased, and the expression of c-Fos in the DNP+GFAP-mCherry group was not significantly different from that in the DNP group. We found that $1.0 \mathrm{mg} / \mathrm{kg} \mathrm{CNO}$ significantly attenuated the cFos expression in DNP+GFAP-M4-mCherry rats compared with DNP+GFAP-mCherry rats (Fig. 4A,B).

Next, we measured the expression of c-Fos after astrocyte activation in naive rats, revealing that it was significantly enhanced by $0.3 \mathrm{mg} / \mathrm{kg}$ CNO in the GFAP-M3-mCherry group compared with the GFAP-mCherry expression group (Fig. $4 A$, $B)$. These results suggested that $\mathrm{MCx}$ astrocytes can modulate the activation of local neurons.

Finally, we assessed the neurochemical properties of these c-Fos-expressing neurons by costaining c-Fos with the excitatory neuronal marker CaMKII and the inhibitory neuronal marker GABA. The majority of neurons expressing c-Fos were colabeled with CaMKII in the MCx region of DNP rats $(>80 \%$ of the c-Fos cells expressed CaMKII), indicating that MCx excitatory neuron activation was promoted by $\mathrm{MCx}$ astrocyte activation. In addition, only a minority of c-Fos-expressing neurons were colabeled with GABA, indicating that they were GABAergic neurons (Fig. 4C); however, the phenotype of the remaining neurons was unknown. Overall, these results indicated that $\mathrm{MCx}$ astrocytes have a regulatory effect on excitatory neurons in the $\mathrm{MCx}$ region.

\section{Chemogenetic inhibition of MCx excitatory neurons reduces mechanical allodynia in rats with DNP}

Considering that regulating the activity of $\mathrm{MCx}$ astrocytes can affect the activation of excitatory neurons localized in the MCx, we next assessed whether inhibiting MCx excitatory neurons would reduce mechanical allodynia in rats with DNP. To address this issue, we injected rats with either the CaMKII-M4-mCherry virus or the CaMKII-mCherry virus bilaterally into the $\mathrm{MCx}$ region after STZ injection; $\sim 4$ weeks after the virus injection, red fluorescence was observed in the MCx region (Fig. 5B-D). Within the virally transduced region, CaMKII-M4-mCherry expression was limited to NeuN (Fig. $5 E$ ), with high penetrance

\section{$\leftarrow$}

with GFAP and did not colocalize with microglia. Scale bars, $100 \mu \mathrm{m}$. $\boldsymbol{H}$, CaMKII-M4-mCherry was expressed in $>91 \%$ of MCX neurons (523 of 547 cells from 5 rats). $I$, Mechanical pain hypersensitivity of rats expressing CaMKII-M4 or CaMKII-mCherry before CNO injection ( $0 \mathrm{~h})$ and over a $6 \mathrm{~h}$ time course after CNO treatment. ${ }^{\# \#} p<0.001$ versus baseline; ${ }^{* *} p<0.01$, ${ }^{* * *} p<0.001$ versus the DNP + CaMKII-mCherry group, $n=10-12$ rats/group, two-way repeated-measures ANOVA followed by Dunnett's multiple comparisons test. J, Maximal possible effects of selective astrocyte inhibition in the MCx at $3 \mathrm{~h}$ after CNO injection on STZinduced mechanical allodynia. ${ }^{* *} p<0.001$ versus the DNP+CaMKII-mCherry group, $n=10-12$ rats/group, two-way repeated-measures ANOVA followed by Dunnett's multiple comparisons test. $\boldsymbol{K}$, The effects of different doses of CNO on mechanical allodynia after STZ injection were evaluated $3 \mathrm{~h}$ after each CNO administration in CaMKII-M4-expressing rats with DNP. ${ }^{\#} p<0.01,{ }^{\# \#} p<0.001$ versus baseline; ${ }^{* * *} p<0.001$ versus the DNP+CaMKII-mCherry group, $n=10$ rats/group, paired-samples $t$ test. L, CaMKII-M4 selective inhibition of neurons in the MCx of rats with DNP attenuates the expression of c-Fos. Scale bars, $100 \mu \mathrm{m}$. Adjacent graph represents the quantification of c-Fos-positive cells. ${ }^{* *} p<0.01$ versus the DNP + CaMKII-mCherry group, $n=5$ or 6 rats/group, Mann-Whitney test.
( $>91 \%$ of NeuN expressed mCherry) (Fig. $5 H$ ), and the noncolocalization of mCherry with GFAP or Ibal demonstrated the specific expression of the CaMKII-M4-mCherry-labeled virus in neurons instead of glia (Fig. $5 F, G$ ).

Three weeks after the CaMKII-M4-mCherry injection, CNO was administered at different doses $(0.03-0.3 \mathrm{mg} / \mathrm{kg}$, i.p.) from days 22 to 28 after STZ injection, and pain was assessed during a $6 \mathrm{~h}$ time course after each administration (Fig. 5A). In the DNP+CaMKII-M4-mCherry group, STZ-induced mechanical allodynia was reversed at $1 \mathrm{~h}$, peaked at $3 \mathrm{~h}$, and remained positive for $5 \mathrm{~h}$ after CNO treatment. However, no significant change in MWT was found in the DNP+CaMKII-mCherry group (Fig. 5I). Compared with DNP+CaMKII-mCherry group, the MWT in the DNP+CaMKII-M4-mCherry group was slightly increased by $0.03 \mathrm{mg} / \mathrm{kg} \mathrm{CNO}$ (without statistical significance), but 0.1 and $0.3 \mathrm{mg} / \mathrm{kg} \mathrm{CNO}$ explicitly increased the threshold (Fig. 5J). The intensity of the effects was also dose-dependent (Fig. $5 K$ ).

After that, we detected the expression of c-Fos in the MCx to prove that CNO specifically inhibited CaMKII-M4-mCherryinfected cells. CNO significantly attenuated c-Fos expression in the DNP+CaMKII-M4-mCherry group compared with the $\mathrm{DNP}+\mathrm{CaMKII}-\mathrm{mCherry}$ group (Fig. 5L). These results suggested that inhibition of MCx excitatory neurons reduces mechanical allodynia in rats with DNP.

\section{Chemogenetic activation of MCx excitatory neurons promotes mechanical allodynia in naive rats}

Previous studies have also shown that MCx excitatory neurons are involved in neuropathic pain (Pagano et al., 2012; Kim et al., 2016; de Andrade et al., 2019), and we further studied whether activation of MCx excitatory neuron is sufficient to induce mechanical allodynia in rats. Naive rats were injected bilaterally with CaMKII-M3-mCherry or CaMKII-mCherry in the MCx region. Three weeks after the virus injection, $\mathrm{CNO}$ was administered at different doses $(0.03-0.3 \mathrm{mg} / \mathrm{kg}$, i.p.) daily from days 22 to 28 , and pain was assessed across a $6 \mathrm{~h}$ time course after each administration (Fig. 6A). In the CaMKII-M3 group, mechanical allodynia was induced gradually, peaked at $2 \mathrm{~h}$, and returned to normal $6 \mathrm{~h}$ after $\mathrm{CNO}$ treatment (Fig. $6 \mathrm{C}$ ). No significant change of MWT was found in the CaMKII-mCherry group (Fig. 6D). Compared with that in the CaMKII-mCherry group, the MWT in the CaMKII-M3+CNO group was slightly decreased with $0.03 \mathrm{mg} / \mathrm{kg}$ CNO (not statistically significant), but strongly decreased with 0.1 and $0.3 \mathrm{mg} / \mathrm{kg} \mathrm{CNO}$ (Fig. $6 E$ ). These results suggested that activation of MCx excitatory neurons can induce mechanical allodynia in naive rats.

Then, we detected the c-Fos expression in the MCx, and the colocalization of c-Fos with mCherry in rats expressing CaMKIIM3 ( $>85 \%$ of the cells expressing c-Fos also expressed mCherry) (Fig. 6B). The immunofluorescent staining showed that CNO significantly increased c-Fos expression in mCherry-expressing neurons in the CaMKII-M3-mCherry group compared with the CaMKII-mCherry group (Fig. 6F). In summary, these results showed that activation of MCx excitatory neurons promotes mechanical allodynia in naive rats.

\section{MCx astrocyte activation in combination with excitatory neuron inhibition did not alter the mechanical allodynia status in naive rats}

To determine the association between MCx excitatory neurons and mechanical allodynia in naive rats following the activation of $\mathrm{MCx}$ astrocytes, we divided the rats into three groups: control, GFAPM3-eGFP+CaMKII-mCherry, and GFAP-M3-eGFP+CaMKII- 
A

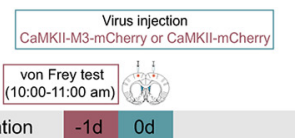

Habituation
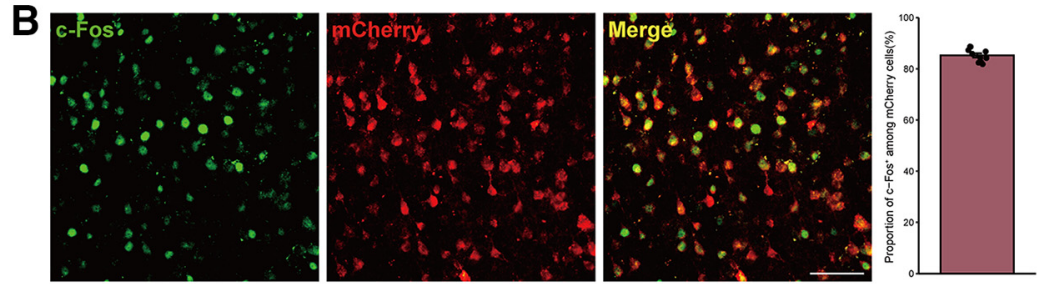

C

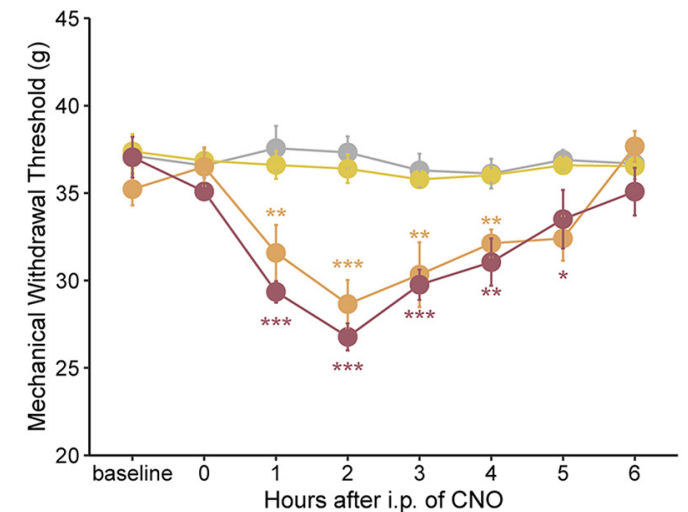

E

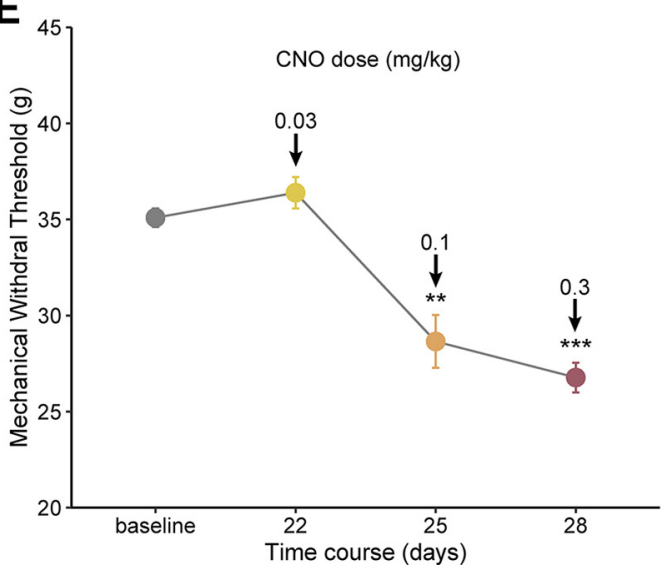
CaMKII-M3-mCherry+CNO $(0.03 \mathrm{mg} / \mathrm{kg})$ CaMKII-M3-mCherry+CNO $(0.1 \mathrm{mg} / \mathrm{kg})$ CaMKII-M3-mCherry+CNO $(0.3 \mathrm{mg} / \mathrm{kg})$
D

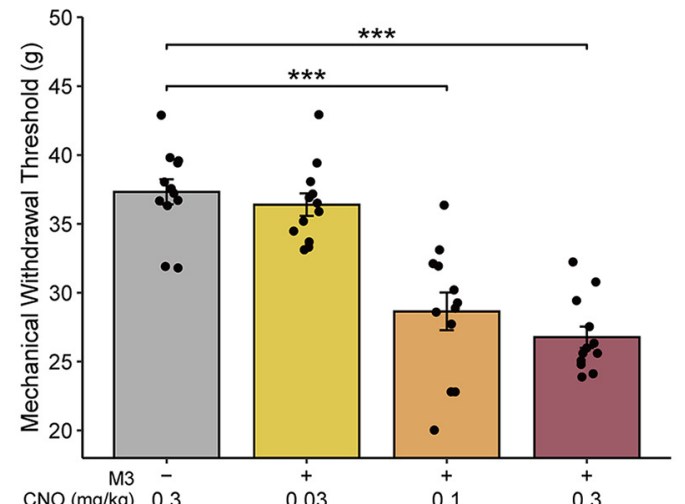

$\mathbf{F}$ CaMKII-mCherry
+ CNO $(0.3 \mathrm{mg} / \mathrm{kg})$

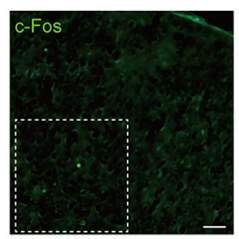
CaMKII-M3-mCherry
+ CNO $(0.3 \mathrm{mg} / \mathrm{kg})$ 0.1 0.3
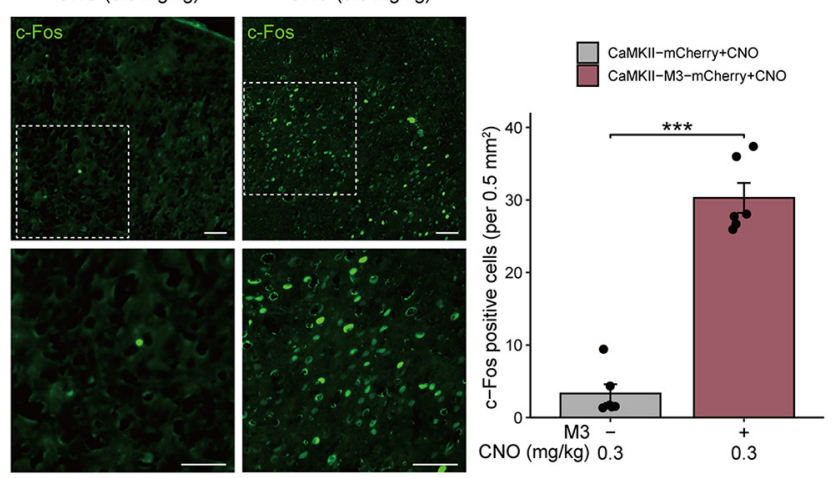

Figure 6. Selective activation of MCx excitatory neurons via CaMKII-M3-mCherry induces mechanical allodynia in normal rats. $\boldsymbol{A}$, Timeline of the experiments. $\boldsymbol{B}$, Photomicrographs illustrating the double immunofluorescence staining of c-Fos (green) and mCherry (red) in the MCx of rats expressing CaMKII-M3-mCherry and treated with CNO. Adjacent graph represents the proportion of c-Fos-positive cells among mCherry-positive cells. C, Mechanical pain hypersensitivities of rats expressing CaMKII-M3-mCherry or CaMKII-mCherry before CNO injection ( 0 h) and over a 6 $\mathrm{h}$ time course after CNO treatment. ${ }^{*} p<0.05,{ }^{* *} p<0.01,{ }^{* * *} p<0.001$ versus the CaMKII-mCherry group, $n=12$ rats/group, two-way repeated-measures ANOVA followed by Dunnett's multiple comparisons test. $D$, Maximal possible effects of selective activation of astrocytes in the $M C x$ at $2 \mathrm{~h}$ after CNO injection on mechanical allodynia in naive rats. *** $p<0.001$ versus the CaMKII-mCherry group, $n=12$ rats/group, two-way repeated-measures ANOVA followed by Dunnett's multiple comparisons test. $\boldsymbol{E}$, The effects of different doses of CNO on mechanical allodynia after virus injection were evaluated $2 \mathrm{~h}$ after each CNO administration in rats expressing CaMKII-M3-mCherry. ${ }^{* *} p<0.01,{ }^{* *} p<0.001$ versus baseline, $n=12$ rats/group, paired-samples $t$ test. $\boldsymbol{F}$, CaMKII-M3-mCherry-induced selective activation of neurons in the MCx of naive rats significantly increased the expression of c-Fos. Scale bars, $100 \mu \mathrm{m}$. Adjacent graph represents the quantification of c-Fos-positive cells. ${ }^{* *} p<0.01$ versus the GFAP-mCherry group, $n=6$ rats/group, Mann-Whitney test.

M4-mCherry. The rats in the latter two groups were injected with GFAP-M3-eGFP combined with CaMKII-mCherry or GFAP-M3eGFP combined with CaMKII-M4-mCherry in the bilateral MCX region. Approximately 4 weeks after virus transfection, green and red fluorescence was shown to be restricted to the MCx region (Fig. $7 B$ ). Following virus expression, a single dose of $\mathrm{CNO}$ was administered via intraperitoneal injection, followed by behavioral testing
(Fig. 7A). CNO injection triggered a significant decrease in the MWT in the GFAP-M3 + CaMKII-mCherry group compared with the control group but did not change the MWT in the GFAPM3+CaMKII-M4-mCherry group (Fig. 7C,D). Therefore, these results demonstrated that $\mathrm{MCx}$ astrocyte activation gives rise to the activation of MCx excitatory neurons, which in turn exacerbates mechanical allodynia in the progression of neuropathic pain. 

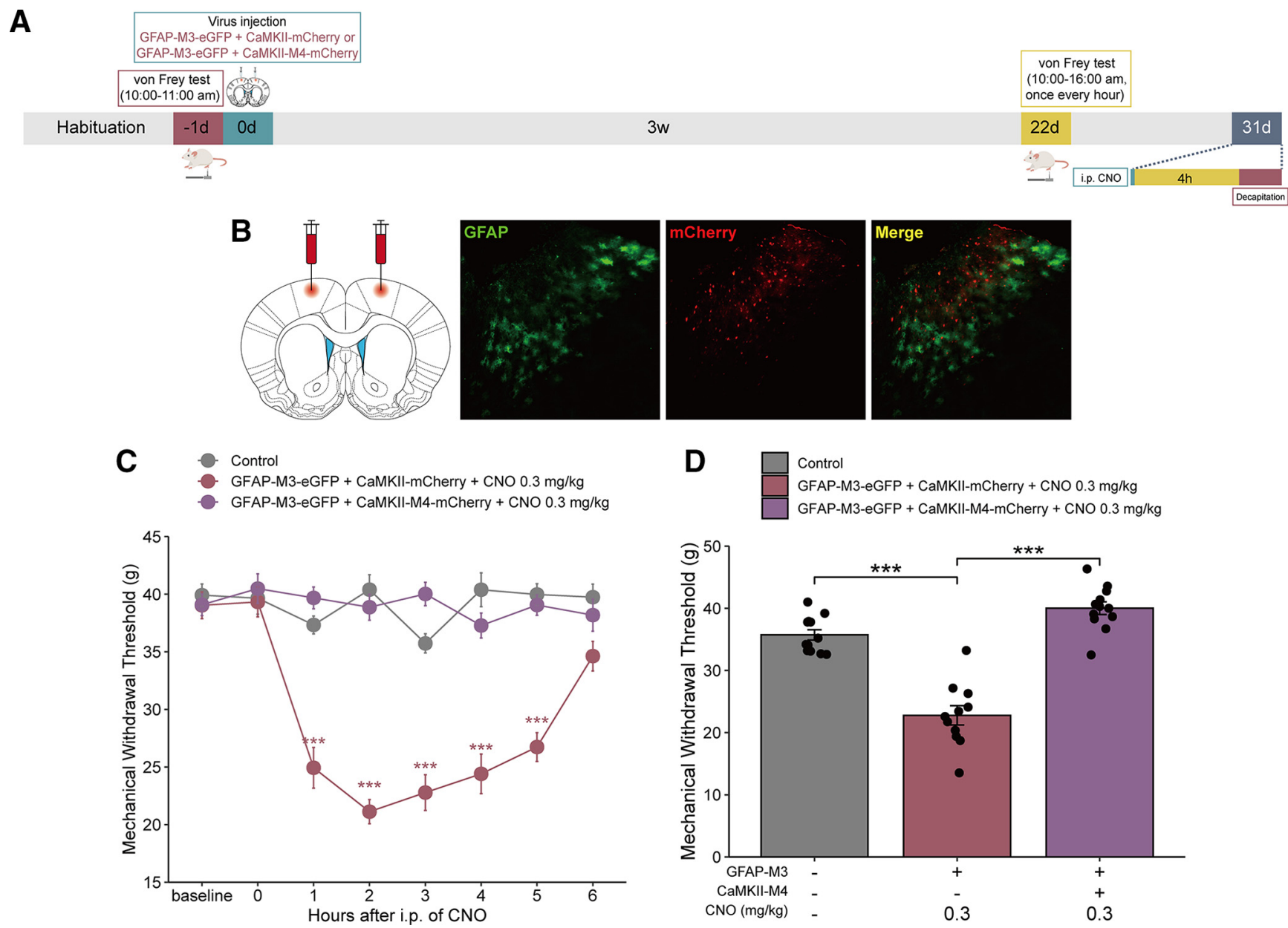

Figure 7. Inhibition of MCx excitatory neurons reverses the mechanical allodynia induced by MCX astrocyte activation in normal rats. $\boldsymbol{A}$, Timeline of the experiments. $\boldsymbol{B}$, The GFAP-M3eGFP + CaMKII-M4-mCherry virus was bilaterally injected into the rat MCX. Green represents the GFAP-M3 virus expression of the eGFP tag. Red represents the CaMKII-M4 virus expression of the mCherry tag. Red-green merged image represents the expression of GFAP-M3-eGFP+CaMKII-M4-mCherry in the MCX. Scale bars, $100 \mu$ m. C, Mechanical pain hypersensitivity of rats expressing GFAP-M3-eGFP+CaMKII-M4-mCherry or GFAP-M3-eGFP+CaMKII-mCherry before CNO injection $(0 \mathrm{~h})$ and over a $6 \mathrm{~h}$ time course after CNO treatment. ${ }^{* * *} p<0.001$ versus the control group, $n=11$ or 12 rats/group, two-way repeated-measures ANOVA followed by Dunnett's multiple comparisons test. $\boldsymbol{D}$, Maximal possible effects of selective activation of astrocytes in the $M C x$ at $2 \mathrm{~h}$ after CNO injection on mechanical allodynia in naive rats. ${ }^{* *} p<0.001$ versus the control group, $n=11$ or 12 rats/group, two-way repeated-measures ANOVA followed by Dunnett's multiple comparisons test.

\section{Effects of inflammatory cytokines on the pain modulatory function of $\mathrm{MCx}$ astrocytes}

Inflammatory cytokines play an essential role in the development of pain. Previous studies have reported that the release of inflammatory cytokines from active astrocytes is the primary cause of DNP (Ji et al., 2014). To investigate whether inflammatory cytokines are involved in pain relief after the modulation of $\mathrm{MCx}$ astrocytes, we used GFAP-M4-mCherry or GFAP-M3-mCherry to inhibit or promote the $\mathrm{MCx}$ astrocyte activity, respectively, and then examined the effects of modulating $\mathrm{MCx}$ astrocyte activity on TNF- $\alpha$ and IL- $\beta$ expression (Fig. 8A). The ELISA results showed that the TNF- $\alpha$ and IL- $\beta$ levels were markedly increased in the MCx of rats with DNP compared with those in the MCx region of control rats, and the expression of TNF- $\alpha$ and IL- $\beta$ in the DNP+GFAP-mCherry group was not significantly different from that in the DNP group. However, compared with the expression in the DNP+GFAP-mCherry group, the TNF- $\alpha$ and IL- $\beta$ expression in the DNP+GFAP-M4mCherry group was significantly decreased. In naive rats, the expression of TNF- $\alpha$ and IL- $\beta$ in the GFAP-mCherry group was not significantly different from that in the control group, and their expression levels in the GFAP-M3-mCherry group were significantly increased compared those in the GFAPmCherry group (Fig. 8B,C). In summary, the activation of $\mathrm{MCX}$ astrocytes may induce and enhance persistent pain states via the release of proinflammatory cytokines.

\section{Discussion}

In this study, we report several novel findings. $\mathrm{MCx}$ astrocytes were regulated by GFAP-M4-mCherry or GFAP-M3-mCherry in rats, and inhibition of $\mathrm{MCx}$ astrocytes via GFAP-M4mCherry attenuated mechanical allodynia in rats with DNP. In contrast, activation of $\mathrm{MCx}$ astrocytes via GFAP-M3-mCherry induced allodynia in naive rats. Moreover, inhibition of $\mathrm{MCx}$ astrocytes via GFAP-M4-mCherry inhibited local MCx neuronal activation and reduced proinflammatory cytokines in rats with DNP. Activation of MCx astrocytes via GFAP-M3-mCherry activated local MCx neuronal activation and induced the release of inflammatory mediators in naive rats. These lines of evidence illustrate that $\mathrm{MCx}$ astrocytes are an integral part of the DNP pathogenesis.

Estrogen levels fluctuate periodically and can affect pain by altering the expression and activities of various neurotransmitters and pain-related receptors (Paredes et al., 2019). In addition, 


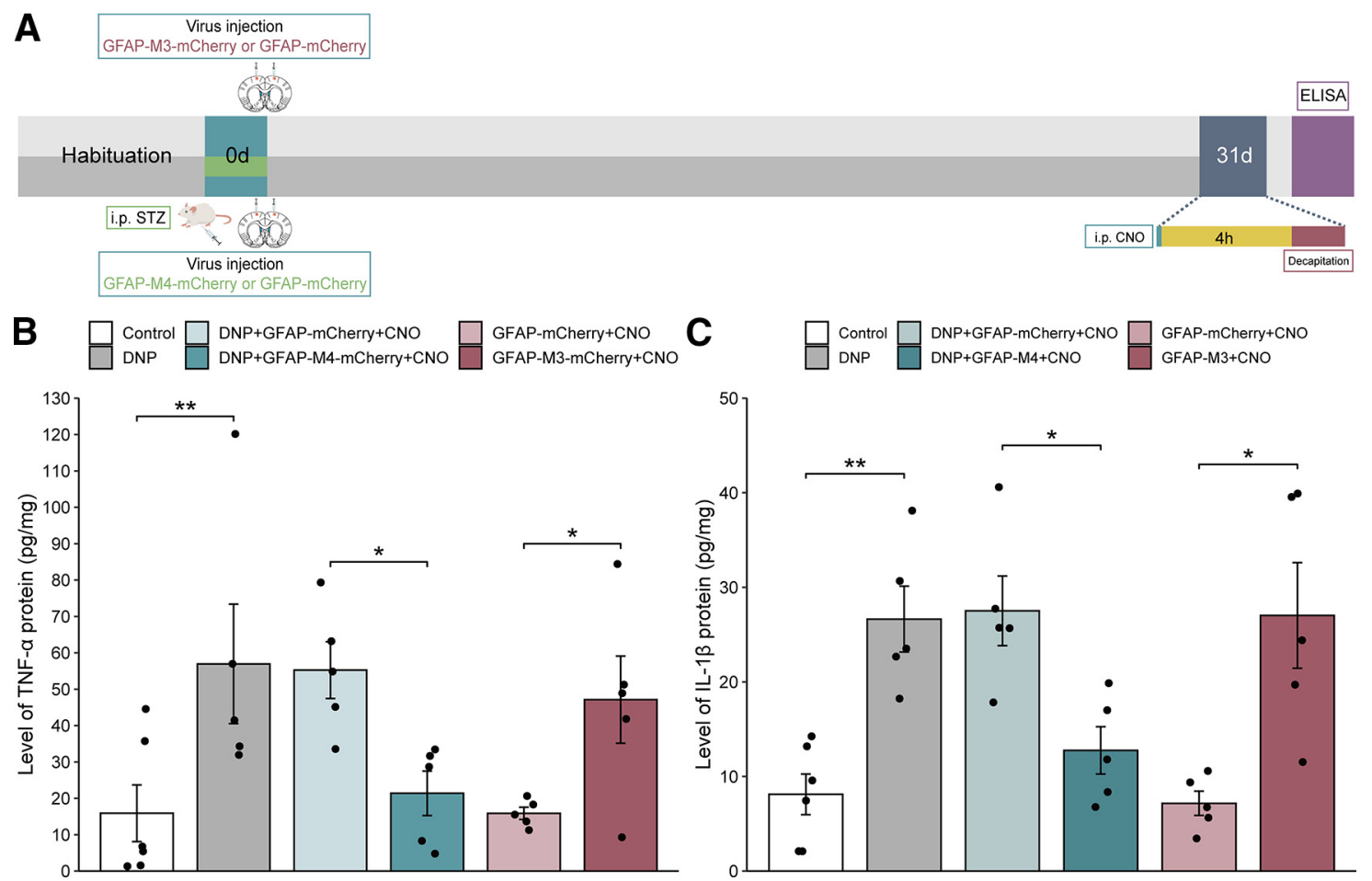

Figure 8. Effects of GFAP-M4-mCherry and GFAP-M3-mCherry on the MCX TNF- $\alpha$ and IL-1 $\beta$ protein levels. A, Timeline of the experiments. $\boldsymbol{B}, \boldsymbol{C}$, The effects of GFAP-M4-mCherry and GFAP-M3-mCherry on the levels of TNF- $\alpha$ and IL- $\beta$ in rats injected with or without STZ. ${ }^{*} p<0.05,{ }^{* *} p<0.01$ versus the control or GFAP-mCherry group, $n=5$ or 6 rats/group, separate one-way ANOVA followed by LSD's multiple comparisons test.

endogenous estrogen can reportedly alleviate the postneuropathic pain response by inhibiting microglial and astrocyte activation (Lee et al., 2018). Therefore, male rats were selected for this study to avoid the potential effect of estrogen on pain.

We used a DREADD approach to specifically manipulate astrocytes in this study because, unlike traditional pharmacologic methods, DREADDs have strong targeting selectivity, can be accurately positioned, and are unconfounded by neuronal off-target effects. The GFAP promoter has been used previously to express proteins in astrocytes but has also been reported to leak into other cells (Fujita et al., 2014). Durkee et al. (2019) demonstrated that GFAP expression was observed in only $\mathrm{GFAP}^{+}$astrocytes and not in neurons of GFAP-M4-expressing rats and concluded that the GFAP promoter was astrocyte-specific. In agreement with this, our data also showed that GFAP was expressed by $\mathrm{GFAP}^{+}$astrocytes but not $\mathrm{NeuN}^{+}$neurons in the $\mathrm{MCx}$ region of rats expressing GFAP-M4.

DREADDs are usually activated by the exogenous ligand CNO; however, a new study recently reported that systemically administered CNO was converted to clozapine, which altered the behavior of non-DREADD-expressing rats (Gomez et al., 2017). This result suggests that it is important to carefully investigate the effects of $\mathrm{CNO}$ in rats. Therefore, our experimental design excluded the effects of $\mathrm{CNO}$ on the mechanical allodynia thresholds in rats, as the GFAP-mCherry group rats were transfected with GFAP-mCherry and exposed to CNO treatment, consistent with the study by Saika et al. (2020). Our results showed that $\mathrm{CNO}$ had no effect on the MWT in rats. We simultaneously investigated the effect of CNO washout on the GFAP signal, which did not different from that at the baseline. In addition, we did not observe motor dysfunction after $\mathrm{CNO}$ administration (data not shown).
In recent decades, the contribution of astrocytes to pain has received considerable attention. A previous study identified a correlation between astrocyte activation and DNP (Feldman et al., 2019). In terms of mechanical allodynia, Liao et al. (2011) highlighted that spinal astrocyte activation promotes the development of mechanical allodynia in rats with DNP. Additionally, numerous studies have shown that inhibiting spinal astrocyte activation can moderate diabetes-induced mechanical allodynia (Dauch et al., 2012; Zuo et al., 2015). These studies all support that astrocyte activation is an integral part of neuropathic pain pathogenesis.

Although the role of astrocytes in pain modulation is well established, most studies have focused on astrocyte reactions in the spinal cord, whereas a supraspinal understanding of the correlation between astrocytes and neuropathy is still limited. There is evidence that astrocytes are also activated in high-level brain regions related to pain processing. In the chronic pain state, astrocyte activation was found concurrently with increased GFAP expression in the thalamus (Panigada and Gosselin, 2011), hippocampus (Guo et al., 2007), periventricular gray matter, and rostral ventromedial medulla (H. Ni et al., 2019). Additionally, in models of neuropathic pain, calcium transients are reportedly enhanced in the cerebral cortex, and inhibiting astrocyte activation reduces synaptic remodeling and palliates pain (Ishikawa et al., 2018; Kummer et al., 2020). The MCx is an important part of the cerebral cortex and is crucial for pain processing. However, the regulatory role of $\mathrm{MCx}$ astrocytes in the pathogenesis of DNP remains to be determined.

In the present study, immunofluorescence analyses showed increased GFAP fluorescence densities within the MCx regions of rats with STZ-induced DNP, which coincides with the reactive pattern of astrocytes. This result is similar to that of Baydas $G$ and Fiore NT, who reported that GFAP expression was increased 
in the cortices and hippocampi of STZ-induced diabetic rats (Baydas et al., 2003; Fiore and Austin, 2019); and differ from those of Lechuga-Sancho et al. (2006), who proposed that the level of GFAP in the diabetic rat hypothalamus was decreased. The discrepancy may be because of the use of different sampling regions. Furthermore, we used a GFAP-M4-mCherry virus to suppress MCx astrocyte activity. We observed that, after the verified inhibition of MCx astrocytes with $\mathrm{CNO}$, the MWT was significantly increased, which was similar to the results observed by Sanada et al. (2014), in the electroacupuncture stimulation of MCX analgesia, indicating that inhibiting MCX astrocytes can effectively alleviate mechanical allodynia in rats with DNP. To further evaluate the effects of MCx astrocytes on mechanical allodynia, we injected the GFAP-M3-mCherry virus into the MCx region of nondiabetic rats. Similar to the results observed by Nam et al. (2016), we observed that the MWT decreased consequently and returned to baseline at $6 \mathrm{~h}$ after intervention, indicating that $\mathrm{MCx}$ astrocyte activation triggered mechanical allodynia in rats. Overall, these findings indicate the potential for astrocytes to serve as a novel therapeutic target for DNP.

A close association exists between astrocytes and neurons. Astrocytes can regulate synaptic transmission by exerting and releasing various neuroactive substances and directly interact with neurons through gap junctions. To better understand the regulatory mechanism of astrocytes in mechanical allodynia and to extend our results to neurons, we observed the expression of the early gene product c-Fos, whose upregulation is correlated with neuronal activation. Upregulated c-Fos expression was found in the MCx region of rats with DNP, which was consistent with the findings reported by Lee et al. (2019). Furthermore, in terms of astrocyte-neuron interactions, $\mathrm{MCx}$ astrocyte inhibition reduced c-Fos expression in the MCx, whereas MCx astrocyte activation increased c-Fos expression in the MCx. Several lines of evidence show that, after nerve injury, astrocytes lose their ability to maintain homeostatic concentrations of extracellular potassium $\left(\mathrm{K}^{+}\right)$and glutamate, leading to neuronal hyperexcitability (Durkee and Araque, 2019; Ji et al., 2019). In our study, the inhibition of MCx excitatory neurons led to reduced mechanical allodynia, which was observed in both STZ-induced rats with DNP and in naive rats whose allodynia was induced by astrocyte activation. These findings suggest that astrocytes adjust neuronal activation to modulate pain responses.

Given that activated astrocytes largely affect neuronal activation under neuropathic pain conditions, it is pivotal to determine the molecular-based mechanisms of astrocytes in neuropathic pain. Numerous experiments have established that the proinflammatory cytokines TNF- $\alpha$ and IL- $1 \beta$ are involved in the regulation of neuropathic pain. Elevated levels of TNF- $\alpha$ (G. L. Ni et al., 2017) and IL-1 $\beta$ (G. L. Ni et al., 2017; Zhou et al., 2018; Jiang et al., 2019) are widely observed in the spinal cords of diabetic rats. Administration of salidroside (G. L. Ni et al., 2017), the anti-inflammatory cytokine IL-35 (Jiang et al., 2019), or minocycline (Pabreja et al., 2011) can reverse high levels of TNF- $\alpha$ and IL- $1 \beta$ induced by DNP, thereby alleviating mechanical allodynia. Excessive concentrations of TNF- $\alpha$ can drastically damage cortical neurons in humans, causing the central inflammation that induces pain. Downregulation of TNF- $\alpha$ and Type 1 receptor expression can noticeably alleviate mechanical allodynia in rats with DNP (Ortmann and Chattopadhyay, 2014). In addition, palliated neuropathic pain was observed in mice overexpressing IL-1 $\beta$ receptor antagonists (Choi et al., 2019), whereas intrathecal administration of IL- $1 \beta$ was shown to induce hyperalgesia (Doyle et al., 2019). The expression of IL-1 $\beta$ is increased dramatically in the spinal activated astrocytes of diabetic mice (Liao et al., 2011), and astrocyte-released IL-1 $\beta$ can directly enhance excitatory synaptic transmission and thus regulate neuronal activation (R. X. Zhang et al., 2008; Gruber-Schoffnegger et al., 2013). Importantly, we also confirmed that MCx astrocyte inhibition could alleviate mechanical allodynia in rats with DNP, which coincided with the inhibition of MCx local neuron excitability and reduced TNF- $\alpha$ and IL- $1 \beta$ expression. These findings indicate that astrocytes may affect neurons by modifying the expression of proinflammatory cytokines and thereby regulate DNP. Overall, our study reveals the potential function of neuron-astrocyte interactions in the development and maintenance of neuropathic pain.

In conclusion, using DREADDs to target and control $\mathrm{MCx}$ astrocytes in rats, we provide compelling evidence to support that MCx astrocytes are crucial mediators of DNP and suggest a potential mechanism for this form of pain; that is, the regulatory effect may be mediated by the release of inflammatory mediators and the activation of excitatory neurons. These results are encouraging and may lead to novel therapies for DNP. These results also establish $\mathrm{MCx}$ astrocytes as promising therapeutic targets for DNP treatment.

\section{References}

Alam U, Sloan G, Tesfaye S (2020) Treating pain in diabetic neuropathy: current and developmental drugs. Drugs 80:363-384.

Baydas G, Reiter RJ, Yasar A, Tuzcu M, Akdemir I, Nedzvetskii VS (2003) Melatonin reduces glial reactivity in the hippocampus, cortex, and cerebellum of streptozotocin-induced diabetic rats. Free Radic Biol Med 35:797-804.

Bönhof GJ, Herder C, Strom A, Papanas N, Roden M, Ziegler D (2019) Emerging biomarkers, tools, and treatments for diabetic polyneuropathy. Endocr Rev 40:153-192.

Chen LM (2018) Cortical representation of pain and touch: evidence from combined functional neuroimaging and electrophysiology in non-human primates. Neurosci Bull 34:165-177.

Choi SR, Han HJ, Beitz AJ, Lee JH (2019) Spinal interleukin-1 $\beta$ inhibits astrocyte cytochrome P450c17 expression which controls the development of mechanical allodynia in a mouse model of neuropathic pain. Front Mol Neurosci 12:153.

Courteix C, Eschalier A, Lavarenne J (1993) Streptozocin-induced diabetic rats: behavioural evidence for a model of chronic pain. Pain 53:81-88.

Dauch JR, Yanik BM, Hsieh W, Oh SS, Cheng HT (2012) Neuron-astrocyte signaling network in spinal cord dorsal horn mediates painful neuropathy of type 2 diabetes. Glia 60:1301-1315.

de Andrade EM, Martinez RC, Pagano RL, Lopes PS, Auada AV, Gouveia FV, Antunes GF, Assis DV, Lebrun I, Fonoff ET (2019) Neurochemical effects of motor cortex stimulation in the periaqueductal gray during neuropathic pain. J Neurosurg 132:239-251.

Doyle TM, Chen Z, Durante M, Salvemini D (2019) Activation of sphingosine-1-phosphate receptor 1 in the spinal cord produces mechanohypersensitivity through the activation of inflammasome and IL-1 $\beta$ pathway. J Pain 20:956-964.

Durkee CA, Araque A (2019) Diversity and specificity of astrocyte-neuron communication. Neuroscience 396:73-78.

Durkee CA, Covelo A, Lines J, Kofuji P, Aguilar J, Araque A (2019) G i/o protein-coupled receptors inhibit neurons but activate astrocytes and stimulate gliotransmission. Glia 67:1076-1093.

Feldman EL, Callaghan BC, Pop-Busui R, Zochodne DW, Wright DE, Bennett DL, Bril V, Russell JW, Viswanathan V (2019) Diabetic neuropathy. Nat Rev Dis Primers 5:42.

Fiore NT, Austin PJ (2019) Peripheral nerve injury triggers neuroinflammation in the medial prefrontal cortex and ventral hippocampus in a subgroup of rats with coincident affective behavioural changes. Neuroscience 416:147-167.

Fox A, Eastwood C, Gentry C, Manning D, Urban L (1999) Critical evaluation of the streptozotocin model of painful diabetic neuropathy in the rat. Pain 81:307-316. 
Fujita T, Chen MJ, Li B, Smith NA, Peng W, Sun W, Toner MJ, Kress BT, Wang L, Benraiss A, Takano T, Wang S, Nedergaard M (2014) Neuronal transgene expression in dominant-negative SNARE mice. J Neurosci 34:16594-16604.

Gomez JL, Bonaventura J, Lesniak W, Mathews WB, Sysa-Shah P, Rodriguez LA, Ellis RJ, Richie CT, Harvey BK, Dannals RF, Pomper MG, Bonci A, Michaelides M (2017) Chemogenetics revealed: DREADD occupancy and activation via converted clozapine. Science 357:503-507.

Grace PM, Strand KA, Galer EL, Urban DJ, Wang X, Baratta MV, Fabisiak TJ, Anderson ND, Cheng K, Greene LI, Berkelhammer D, Zhang Y, Ellis AL, Yin HH, Campeau S, Rice KC, Roth BL, Maier SF, Watkins LR (2016) Morphine paradoxically prolongs neuropathic pain in rats by amplifying spinal NLRP3 inflammasome activation. Proc Natl Acad Sci USA 113:E3441-E3450.

Gruber-Schoffnegger D, Drdla-Schutting R, Honigsperger C, Wunderbaldinger G, Gassner M, Sandkuhler J (2013) Induction of thermal hyperalgesia and synaptic long-term potentiation in the spinal cord lamina I by TNF- and IL1 is mediated by glial cells. J Neurosci 33:6540-6551.

Guo W, Wang H, Watanabe M, Shimizu K, Zou S, LaGraize SC, Wei F, Dubner R, Ren K (2007) Glial-cytokine-neuronal interactions underlying the mechanisms of persistent pain. J Neurosci 27:6006-6018.

Ishikawa T, Eto K, Kim SK, Wake H, Takeda I, Horiuchi H, Moorhouse AJ, Ishibashi H, Nabekura J (2018) Cortical astrocytes prime the induction of spine plasticity and mirror image pain. Pain 159:1592-1606.

Ji RR, Xu ZZ, Gao YJ (2014) Emerging targets in neuroinflammation-driven chronic pain. Nat Rev Drug Discov 13:533-548.

Ji RR, Chamessian A, Zhang YQ (2016) Pain regulation by non-neuronal cells and inflammation. Science 354:572-577.

Ji RR, Donnelly CR, Nedergaard M (2019) Astrocytes in chronic pain and itch. Nat Rev Neurosci 20:667-685.

Jiang Y, Wang J, Li H, Xia L (2019) IL-35 alleviates inflammation progression in a rat model of diabetic neuropathic pain via inhibition of JNK signaling. J Inflamm (Lond) 16:19.

Kim J, Ryu SB, Lee SE, Shin J, Jung HH, Kim SJ, Kim KH, Chang JW (2016) Motor cortex stimulation and neuropathic pain: how does motor cortex stimulation affect pain-signaling pathways? J Neurosurg 124:866-876.

Kragel PA, Kano M, Van Oudenhove L, Ly HG, Dupont P, Rubio A, DelonMartin C, Bonaz BL, Manuck SB, Gianaros PJ, Ceko M, Reynolds Losin EA, Woo CW, Nichols TE, Wager TD (2018) Generalizable representations of pain, cognitive control, and negative emotion in medial frontal cortex. Nat Neurosci 21:283-289.

Kummer KK, Mitrić M, Kalpachidou T, Kress M (2020) The medial prefrontal cortex as a central hub for mental comorbidities associated with chronic pain. Int J Mol Sci 21:3440.

Lechuga-Sancho AM, Arroba AI, Frago LM, García-Cáceres C, de Célix AD, Argente J, Chowen JA (2006) Reduction in the number of astrocytes and their projections is associated with increased synaptic protein density in the hypothalamus of poorly controlled diabetic rats. Endocrinology 147:5314-5324

Lee JY, Choi HY, Ju BG, Yune TY (2018) Estrogen alleviates neuropathic pain induced after spinal cord injury by inhibiting microglia and astrocyte activation. Biochim Biophys Acta Mol Basis Dis 1864:2472-2480.

Lee JY, Choi HY, Park CS, Pyo MK, Yune TY, Kim GW, Chung SH (2019) GS-KG9 ameliorates diabetic neuropathic pain induced by streptozotocin in rats. J Ginseng Res 43:58-67.

Li T, Chen X, Zhang C, Zhang Y, Yao W (2019) An update on reactive astrocytes in chronic pain. J Neuroinflammation 16:140.

Liao YH, Zhang GH, Jia D, Wang P, Qian NS, He F, Zeng XT, He Y, Yang YL, Cao DY, Zhang Y, Wang DS, Tao KS, Gao CJ, Dou KF (2011) Spinal astrocytic activation contributes to mechanical allodynia in a mouse model of type 2 diabetes. Brain Res 1368:324-335.

Mitrirattanakul S, Ramakul N, Guerrero AV, Matsuka Y, Ono T, Iwase H, Mackie K, Faull KF, Spigelman I (2006) Site-specific increases in peripheral cannabinoid receptors and their endogenous ligands in a model of neuropathic pain. Pain 126:102-114.
Nam Y, Kim JH, Kim JH, Jha MK, Jung JY, Lee MG, Choi IS, Jang IS, Lim DG, Hwang SH, Cho HJ, Suk K (2016) Reversible induction of pain hypersensitivity following optogenetic stimulation of spinal astrocytes. Cell Rep 17:3049-3061.

Ni GL, Cui R, Shao AM, Wu ZM (2017) Salidroside ameliorates diabetic neuropathic pain in rats by inhibiting neuroinflammation. J Mol Neurosci 63:9-16.

Ni H, Xu LS, Wang Y, Li H, An K, Liu M, Liu Q, Deng H, He Q, Huang B, Fang J, Yao M (2019) Astrocyte activation in the periaqueductal gray promotes descending facilitation to cancer-induced bone pain through the JNK MAPK signaling pathway. Mol Pain 15:1744806919831909.

Ortmann KL, Chattopadhyay M (2014) Decrease in neuroimmune activation by HSV-mediated gene transfer of TNF $\alpha$ soluble receptor alleviates pain in rats with diabetic neuropathy. Brain Behav Immun 41:144-151.

Pabreja K, Dua K, Sharma S, Padi SS, Kulkarni SK (2011) Minocycline attenuates the development of diabetic neuropathic pain: possible antiinflammatory and anti-oxidant mechanisms. Eur J Pharmacol 661:15-21.

Pagano RL, Fonoff ET, Dale CS, Ballester G, Teixeira MJ, Britto LR (2012) Motor cortex stimulation inhibits thalamic sensory neurons and enhances activity of PAG neurons: possible pathways for antinociception. Pain 153:2359-2369.

Panigada T, Gosselin RD (2011) Behavioural alteration in chronic pain: are brain glia involved? Med Hypotheses 77:584-588.

Paredes S, Cantillo S, Candido KD, Knezevic NN (2019) An association of serotonin with pain disorders and its modulation by estrogens. Int J Mol Sci 20:5729.

Peltier A, Goutman SA, Callaghan BC (2014) Painful diabetic neuropathy. BMJ 348:g1799.

Rajchgot T, Thomas SC, Wang JC, Ahmadi M, Balood M, Crosson T, Dias JP, Couture R, Claing A, Talbot S (2019) Neurons and microglia: a sicklysweet duo in diabetic pain neuropathy. Front Neurosci 13:25

Rosenberger DC, Blechschmidt V, Timmerman H, Wolff A, Treede RD (2020) Challenges of neuropathic pain: focus on diabetic neuropathy. J Neural Transm (Vienna) 127:589-624

Saika F, Matsuzaki S, Kobayashi D, Ideguchi Y, Nakamura TY, Kishioka S, Kiguchi N (2020) Chemogenetic regulation of CX3CR1-expressing microglia using Gi-DREADD exerts sex-dependent anti-allodynic effects in mouse models of neuropathic pain. Front Pharmacol 11:925.

Sanada LS, Sato KL, Machado NL, Carmo E, Sluka KA, Fazan VP (2014) Cortex glial cells activation, associated with lowered mechanical thresholds and motor dysfunction, persists into adulthood after neonatal pain. Int J Dev Neurosci 35:55-63.

Scholz J, Woolf CJ (2007) The neuropathic pain triad: neurons, immune cells and glia. Nat Neurosci 10:1361-1368.

Tsubokawa T, Katayama Y, Yamamoto T, Hirayama T, Koyama S (1991) Chronic motor cortex stimulation for the treatment of central pain. Acta Neurochir Suppl (Wien) 52:137-139.

Vanderwall AG, Milligan ED (2019) Cytokines in pain: harnessing endogenous anti-inflammatory signaling for improved pain management. Front Immunol 10:3009.

Zhang RX, Li A, Liu B, Wang L, Ren K, Zhang H, Berman BM, Lao L (2008) IL-1ra alleviates inflammatory hyperalgesia through preventing phosphorylation of NMDA receptor NR-1 subunit in rats. Pain 135:232-239.

Zhang ZJ, Jiang BC, Gao YJ (2017) Chemokines in neuron-glial cell interaction and pathogenesis of neuropathic pain. Cell Mol Life Sci 74:32753291.

Zhou R, Xu T, Liu X, Chen Y, Kong D, Tian H, Yue M, Huang D, Zeng J (2018) Activation of spinal dorsal horn P2Y13 receptors can promote the expression of IL-1 $\beta$ and IL- 6 in rats with diabetic neuropathic pain. J Pain Res 11:615-628.

Zuo ZF, Liao YH, Ding T, Dong YL, Qu J, Wang J, Wei YY, Lu YC, Liu XZ, Li YQ (2015) Astrocytic NDRG2 is involved in glucocorticoid-mediated diabetic mechanical allodynia. Diabetes Res Clin Pract 108:128-136. 